\title{
Regularized Discriminant Analysis: A Large Dimensional Study
}

Thesis by

Xiaoke Yang

In Partial Fulfillment of the Requirements

For the Degree of

Masters of Science

King Abdullah University of Science and Technology

Thuwal, Kingdom of Saudi Arabia

April, 2018 


\section{EXAMINATION COMMITTEE PAGE}

The thesis of Xiaoke Yang is approved by the examination committee

Committee Chairperson: Tareq Al-Naffouri

Committee Member: Mohamed-Slim Alouini

Committee Member: Xiangliang Zhang 
(C)April, 2018

Xiaoke Yang

All Rights Reserved 


\section{ABSTRACT \\ Regularized Dsiscriminant Analysis: A Large Dimensional Study Xiaoke Yang}

In this thesis, we focus on studying the performance of general regularized discriminant analysis (RDA) classifiers. The data used for analysis is assumed to follow Gaussian mixture model with different means and covariances. RDA offers a rich class of regularization options, covering as special cases the regularized linear discriminant analysis (RLDA) and the regularized quadratic discriminant analysis (RQDA) classifiers. We analyze RDA under the double asymptotic regime where the data dimension and the training size both increase in a proportional way. This double asymptotic regime allows for application of fundamental results from random matrix theory. Under the double asymptotic regime and some mild assumptions, we show that the asymptotic classification error converges to a deterministic quantity that only depends on the data statistical parameters and dimensions. This result not only implicates some mathematical relations between the misclassification error and the class statistics, but also can be leveraged to select the optimal parameters that minimize the classification error, thus yielding the optimal classifier. Validation results on the synthetic data show a good accuracy of our theoretical findings. We also construct a general consistent estimator to approximate the true classification error in consideration of the unknown previous statistics. We benchmark the performance of our proposed consistent estimator against classical estimator on synthetic data. The observations demonstrate that the general estimator outperforms others in terms of mean squared error (MSE). 


\section{ACKNOWLEDGEMENTS}

First, I would like to express my thanks to KAUST, a world-class and promising university providing me a chance to start my master life.

Next, I would really appreciate my advisor, Professor Tareq Al-Naffouri, who always takes good care of both my study and life. His encouragement and guidance are the source for my confidence to continue pursuing my research career.

I would also like to thank khalil, a preeminent advanced phD senior, for his step-

by-step instruction on my research process. Also for his precise feedback and high participation in my first accepted paper. I would also express my gratitude to Dr.Abla Kammoun for her expert opinion and professional suggestions on my paper revise.

I will also acknowledgement my friends and colleagues in our lab, Osman, Ayed, Chen, Xing, Suliman and khalil. They are sources of happiness for my life in KAUST.

Finally, I would express my sincere gratitude to my boyfriend, guolei sun. He has always accompanied me to overcome all the difficulties I encountered in study and life. He always gives me endless support and concern to pursue my ideal life. 


\section{TABLE OF CONTENTS}

\begin{tabular}{ll}
\hline Examination Committee Page & 2
\end{tabular}

\begin{tabular}{ll}
\hline Copyright & 3
\end{tabular}

\begin{tabular}{|ll}
\hline Abstract & 4
\end{tabular}

\begin{tabular}{|l|l|}
\hline Acknowledgements & 5
\end{tabular}

\begin{tabular}{lr}
\hline List of Figures & 9
\end{tabular}

$\begin{array}{lll}1 & \text { Introduction } & 10\end{array}$

$1.1 \quad$ Discriminant Analysis for Classification . . . . . . . . . . . . . . . . . 10

1.2 Previous Work . . . . . . . . . . . . . . . . . . . . . . . . . . 11

1.3 Objectives and Contributions . . . . . . . . . . . . . . . . 12

1.4 Notations . . . . . . . . . . . . . . . . . . . 13

$\begin{array}{lll}2 & \text { RDA Classifier for Binary Classification } & 14\end{array}$

$\begin{array}{lll}3 & \text { Main Results } & 18\end{array}$

3.1 Technical Assumptions . . . . . . . . . . . . . . . . . . . . . . . . . 18

3.2 Central Limit Theorem(CLT) . . . . . . . . . . . . . . . . . . . . . . 19

3.3 Deterministic Equivalent . . . . . . . . . . . . . . . . . . . . 20

3.4 Validation with Synthetic Data . . . . . . . . . . . . . . . . 24

4 General Consistent Estimator for RDA 26

4.1 General Consistent Estimator of the Testing Error . . . . . . . . . . . 26

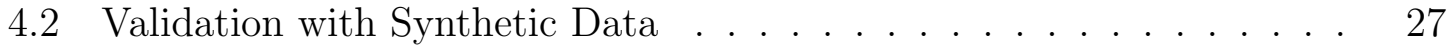

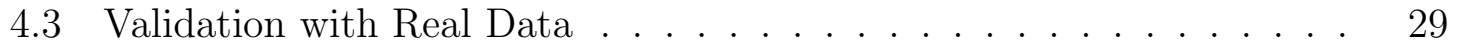

5 Concluding Remarks

5.1 Summary . . . . . . . . . . . . . . . . . . . . 31

5.2 Future Research Work . . . . . . . . . . . . . . . . . . . . . . . . . . 31 
\begin{tabular}{ll}
\hline References & 32
\end{tabular}

\begin{tabular}{|ll}
\hline Appendices & 34
\end{tabular}

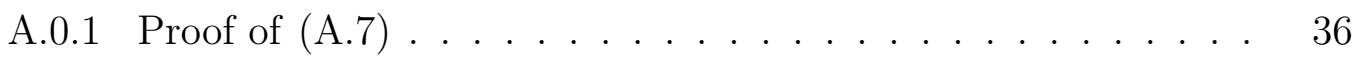

A.0.2 Proof of $(\mathrm{A} .80] \ldots \ldots \ldots \ldots \ldots \ldots \ldots \ldots$

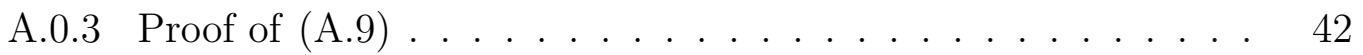

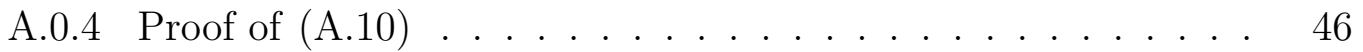

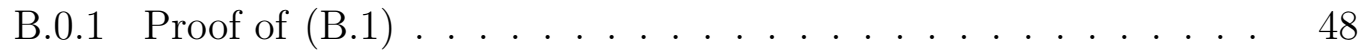

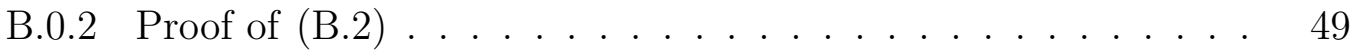

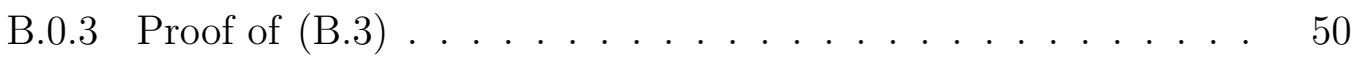




\section{LIST OF FIGURES}

3.1 RDA classifier performance in terms of classification error with equal training, $n_{0}=n_{1}$. The $x$ axis is the number of the data dimension. . . 25

3.2 Comparison of performance of RDA and R-LDA in terms of classification error with equal training, $n_{0}=n_{1}$. The $x$ axis is regularization parameter $\lambda$ and $y$ axis is regularization parameter $\gamma$ for $p \in\{300,400,500\}$ and $c=1$. . . . . . . . . . . . . . 25

4.1 RDA general consistent estimator performance in terms of classification error with equal training, $n_{0}=n_{1}$. The $x$ axis is the number of the data dimension. . . . . . . . . . . . . . . . . . . . . . . . 29

4.2 MSE performance of the proposed general consistent estimators compared with 5 -fold cross validation and plug in estimator. . . . . . . . 29

4.3 MSE performance of the proposed general consistent estimators compared with 5-fold cross validation and plug in estimator. We choose equal training $n_{0}=n_{1}$. . . . . . . . . . . . . . . . . . . . . . 30 


\section{Chapter 1}

\section{Introduction}

\subsection{Discriminant Analysis for Classification}

Many classification approaches are studied to solve the classification problems in today's hot pattern recognition and machine learning fields[1]. Among them, we distinguish discriminant analysis, which are extensively used in a large panel of applications, such as bioinformatics [2] and finance [3] to name a few. Belonging to the larger set of model-based classification methods, their popularity owes to the fact that they rely on probabilistic foundations, making them optimal under the assumptions they have been built upon. Linear discriminant analysis (LDA) and Quadratic discriminant analysis (QDA) are the most typical cases of discriminant analysis. Both of them are based on the assumption that data from each class are drawn from a specific Gaussian distribution. The single difference lies in that LDA assumes the same covariance matrix for all classes, while QDA allows different covariance matrices. Under the Gaussian assumption and assuming the knowledge of the mean and

covariance matrices for each class, LDA and QDA produce the classifiers that minimize the mis-classification error rate.

This high performance is, however, in practice not always guaranteed. The major reason for that is related to the fact that the means and covariance matrices associated with each class could not be perfectly acquired. They can instead be estimated based on available training sets for which the class labels are given. This results in performance losses which become all the more large when the sample size for each class 
is small with respect to their dimension, causing the estimated covariance matrices to be highly inaccurate and ill-conditioned. One approach to get around this issue, which dates back to the early works of Friedman [4], is to regularize the covariance matrix. The regularization artifice serves to improve the stability of these estimates by shrinking them towards the identity matrix. This can be accomplished by employing one regularization parameter in LDA or QDA, giving rise to what is known as regularized LDA (R-LDA) and regularized QDA (R-QDA). A better flexibility can be obtained by using two regularization parameters offering a better regulation of the weights associated with samples of each class. This approach, which seemingly can bring better performances, is known as regularized discriminant analysis (RDA).

\subsection{Previous Work}

A lot of attention has been devoted to analyzing the performances of R-LDA and R-QDA classifiers in recent studies. One frequently used approach is to suppose the data dimension fixed while the the training size increases to infinity $(n \rightarrow \infty)$. Under this setting, the work in [5] has successfully derived the close form solution for misclassification error in QDA. However, this traditional approach will not be helpful in cases where the data dimension is comparable to the training size. More importantly, this approach cannot generalize to our study for RDA. So we need a different regime to carry out the RDA analysis. Another frequently used regime, is the double asymptotic regime in which the number of samples and their dimensions grow large with the same pace. Double asymptotic regime has been popular in recent studies. The first application of this double asymptotic regime can be dated back to the study in [6]. The interest of this regime lies in that it allows leveraging a large body of results from the theory of random matrices, opening up possibilities of accurate characterization of the asymptotic misclassification error rate. Towards this goal, the work in [7] studies the asymptotic misclassification error rate of the R-LDA, 
while the R-QDA has only recently been analyzed in [8]. The work in [8] presents the exact solution for misclassification error of R-QDA and indicates some special cases in which either R-LDA or R-QDA should give better performance. To the best of the authors' knowledge, the RDA, which should offer better capabilities, has not been theoretically studied. The present work aims to fill this gap.

\subsection{Objectives and Contributions}

Restricting our attention to binary classification, we analyse the asymptotic misclassification error rate for the RDA. In particular, we identify sufficient assumptions on the distance between covariance matrices and means of both classes ensuring non-trivial classification error rate. Under these assumptions, we show that the misclassification error rate can be approximated in the asymptotic regime by deterministic quantities that depend solely on the samples' size and their dimensions as well as the means and covariances of each class. The major interests behind this result are twofold. First, it allows to enlighten the impact of the intervening parameters on the classification performances. Second, it can be used in practice to properly tune the regularization parameters, so as to reap the full potential of RDA. In a nutshell, the contributions are summarized as follows

- Under some mild assumptions, we show that the classification error rate approaches a non-trivial deterministic quantity that only depends on the classes' statistics and the problem's dimension.

- Based on the derived deterministic classification error, we illustrate the importance of properly selecting the pair of parameters that minimizes the misclassification error rate.

- We construct a general consistent estimator for approximating the misclassification error. 
- We validate the theoretical results using synthetic data and demonstrate the accuracy of our findings for deterministic equivalent misclassification error and constructed estimator performance.

\subsection{Notations}

Throughout this thesis, we use non-boldface lowercase letters to denote scalars, boldface lowercase letters to denote vectors and boldface uppercase letters to denote matrices. $\mathbf{I}_{p}$ denotes the $p$ dimensional identity matrix. $\mathbf{1}_{p \times n}$ denotes the $p$ by $n$ matrix with all entries being one. $\|$.$\| denotes the Euclidean norm for vectors and the spectral$ norm for matrices. (.. $)^{T}, \operatorname{tr}($.$) and |.| respectively denote the transpose, the trace and$ the determinant of a matrix. For two functionals $f$ and $g$, we say that $f=\mathcal{O}(g)$, if $\exists 0<M<\infty$ such that $|f| \leq M|g| . \mathbb{P}($.$) denotes the probability measure. \stackrel{p}{\rightarrow}$ denotes the convergence in probability. $\stackrel{a . s .}{\longrightarrow}$ denotes the almost sure convergence of random variables. $\Phi(x)=\int_{-\infty}^{x} \frac{\exp \left(-\frac{t^{2}}{2}\right)}{\sqrt{2 \pi}} d t$ denotes the cumulative density function (CDF) of the standard normal distribution.

The rest of this thesis is organized as follows: Chapter 2 introduces the RDA problem. Chapter 3 first exposes the assumptions and the theoretical findings and presents the validation results for accuracy of our derived results. The detailed proofs for the theorems can be found in the appendix. Chapter 4 gives the construction for general consistent estimator and evaluates its performance. Section Finally, we make a conclusion for this thesis. 


\section{Chapter 2}

\section{RDA Classifier for Binary Classification}

We consider the binary classification problem in which we aim to assign an observation $\mathbf{x} \in \mathbb{R}^{p}$ to the class $\mathcal{C}_{i}, i \in\{0,1\}$ which $\mathbf{x}$ most likely belongs to. We assume that observations vectors sampled from class $\mathcal{C}_{i}, i \in\{0,1\}$ follow a multivariate Gaussian distribution with mean $\boldsymbol{\mu}_{i} \in \mathbb{R}^{p}$ and covariance $\boldsymbol{\Sigma}_{i} \in \mathbb{R}^{p \times p}$. Denote the prior probability of $\mathbf{x}$ belonging to class $\mathcal{C}_{i}$ by $\pi_{i}, i \in\{0,1\}$. The Bayes classifier is thus the one that maximizes the posterior probability [9], which boils down to selecting the class that corresponds to the highest classification score $\delta_{i}^{R D A}(\mathbf{x}), i \in\{0,1\}$ where

$$
\delta_{i}^{R D A}(\mathbf{x})=-\frac{1}{2} \log \left|\boldsymbol{\Sigma}_{i}\right|-\frac{1}{2}\left(\mathbf{x}-\mu_{i}\right)^{T} \boldsymbol{\Sigma}_{i}^{-1}\left(\mathbf{x}-\mu_{i}\right)+\log \pi_{i} .
$$

More formally, we assign $\mathbf{x}$ to class $i^{*}$ where

$$
i^{*}=\underset{i \in\{0,1\}}{\arg \max } \delta_{i}^{R D A}(\mathbf{x}) .
$$

The discriminant function $\delta_{i}^{R D A}(\mathbf{x})$ involves the exact statistics of each class, namely, their associated mean vectors and covariances. In practice, these parameters could not be perfectly known beforehand. They are estimated using available training data. We assume that $n_{i}, i \in\{0,1\}$ independent training samples are provided for class $\mathcal{C}_{i}$, respectively denoted by $\mathcal{S}_{0}=\left\{\mathbf{x}_{l} \in \mathcal{C}_{0}\right\}_{l=1}^{n_{0}}$ and $\mathcal{S}_{1}=\left\{\mathbf{x}_{l} \in \mathcal{C}_{1}\right\}_{l=n_{0}+1}^{n_{0}+n_{1}=n}$. Based on this training data, we consider the following sample estimates of the mean and covariance 
matrices

$$
\begin{aligned}
& \overline{\mathbf{x}}_{i}=\frac{1}{n_{i}} \sum_{l \in \mathcal{T}_{i}} \mathbf{x}_{l}, \quad i \in\{0,1\} . \\
& \widehat{\mathbf{\Sigma}}_{i}=\frac{1}{n_{i}-1} \sum_{l \in \mathcal{T}_{i}}\left(\mathbf{x}_{l}-\overline{\mathbf{x}}_{i}\right)\left(\mathbf{x}_{l}-\overline{\mathbf{x}}_{i}\right)^{T}, \quad i \in\{0,1\} \\
& \widehat{\mathbf{\Sigma}}=\frac{n_{0}-1}{n-2} \widehat{\boldsymbol{\Sigma}}_{0}+\frac{n_{1}-1}{n-2} \widehat{\mathbf{\Sigma}}_{1},
\end{aligned}
$$

where $\widehat{\boldsymbol{\Sigma}}$ is the pooled sample covariance. LDA and QDA classifiers are respectively obtained when $\widehat{\boldsymbol{\Sigma}}$ or $\widehat{\boldsymbol{\Sigma}}_{i}$ are used in place of $\boldsymbol{\Sigma}_{i}, i \in\{0,1\}$. They can be viewed as extreme cases of the RDA which consists in using the following regularized covariance matrix

$$
\widehat{\boldsymbol{\Sigma}}_{i}(\lambda)=\frac{(1-\lambda) n_{i} \widehat{\boldsymbol{\Sigma}}_{i}+\lambda n \widehat{\boldsymbol{\Sigma}}}{(1-\lambda) n_{i}+\lambda n}, i \in\{0,1\}
$$

where $\lambda$ controls the shrinkage of the individual covariance of QDA toward the pooled covariance matrix in LDA. In this manner, they define a parametrized class of discriminant analysis classifiers ranging from $\operatorname{LDA}(\lambda=1)$ to $\mathrm{QDA}(\lambda=0)$. If the training size is much lower than the data dimension, $\widehat{\boldsymbol{\Sigma}}_{i}(\lambda)$ is singular. Singularity concerns will thus arise if it is directly plugged in $\delta_{i}^{R D A}(\mathbf{x})$ in place of $\boldsymbol{\Sigma}_{i}$. To overcome these issues, a common way is to use a second regularization parameter $\gamma \in[0,1]$ aiming to shrinking $\widehat{\boldsymbol{\Sigma}}_{i}(\lambda)$ towards the identity matrix. The regularized covariance matrix that is used in RDA takes

$$
\widehat{\boldsymbol{\Sigma}}_{i}(\lambda, \gamma)=\gamma \frac{(1-\lambda) n_{i} \widehat{\boldsymbol{\Sigma}}_{i}+\lambda n \widehat{\boldsymbol{\Sigma}}}{(1-\lambda) n_{i}+\lambda n}+(1-\gamma) \mathbf{I}_{p}
$$


Defining the following quantities

$$
\begin{aligned}
\mathbf{H}_{0} & =\left[(1-\gamma) \mathbf{I}_{p}+\alpha_{0} \widehat{\boldsymbol{\Sigma}}_{0}+\beta_{0} \widehat{\boldsymbol{\Sigma}}_{1}\right]^{-1} . \\
\alpha_{0} & =\frac{n_{0} \gamma}{n_{0}+\lambda n_{1}}, \beta_{0}=\frac{n_{1} \gamma \lambda}{n_{0}+\lambda n_{1}} . \\
\mathbf{H}_{1} & =\left[(1-\gamma) \mathbf{I}_{p}+\alpha_{1} \widehat{\Sigma}_{0}+\beta_{1} \widehat{\boldsymbol{\Sigma}}_{1}\right]^{-1} . \\
\alpha_{1} & =\frac{n_{0} \gamma \lambda}{n_{1}+\lambda n_{0}}, \beta_{1}=\frac{n_{1} \gamma}{n_{1}+\lambda n_{0}}
\end{aligned}
$$

the discriminant rule in 2.1 for RDA simplifies to

$$
\widehat{\delta}_{i}^{R D A}(\mathbf{x})=\frac{1}{2} \log \left|\mathbf{H}_{i}\right|-\frac{1}{2}\left(\mathbf{x}-\overline{\mathbf{x}}_{i}\right)^{T} \mathbf{H}_{i}\left(\mathbf{x}-\overline{\mathbf{x}}_{i}\right)+\log \pi_{i}
$$

Conditioning on the training samples $\mathcal{S}_{i}, i \in\{0,1\}$, the conditional misclassification error rate associated to class $\mathcal{C}_{i}$ is given by

$$
\epsilon_{i}^{R D A}=\mathbb{P}\left[(-1)^{i} \widehat{\delta}_{0}^{R D A}(\mathbf{x})<(-1)^{i} \widehat{\delta}_{1}^{R D A}(\mathbf{x}) \mid \mathbf{x} \in \mathcal{C}_{i}\right]
$$

Thus, the total misclassification error rate writes as

$$
\epsilon^{R D A}=\pi_{0} \epsilon_{0}^{R D A}+\pi_{1} \epsilon_{1}^{R D A}
$$

It is also important to note that using some basic manipulations, the conditional misclassification error rate can be written as

$$
\epsilon_{i}^{R D A}=\mathbb{P}\left[\boldsymbol{\omega}^{T} \mathbf{B}_{i} \boldsymbol{\omega}+2 \boldsymbol{\omega}^{T} \mathbf{y}_{i}<\xi_{i} \mid \boldsymbol{\omega} \sim \mathcal{N}\left(\mathbf{0}, \mathbf{I}_{p}\right)\right], i \in\{0,1\}
$$


where

$$
\begin{aligned}
\mathbf{B}_{i} & =\boldsymbol{\Sigma}_{i}^{1 / 2}\left(\mathbf{H}_{1}-\mathbf{H}_{0}\right) \boldsymbol{\Sigma}_{i}^{1 / 2} \\
\mathbf{y}_{i} & =\boldsymbol{\Sigma}_{i}^{1 / 2}\left[\mathbf{H}_{1}\left(\boldsymbol{\mu}_{i}-\overline{\mathbf{x}}_{1}\right)-\mathbf{H}_{0}\left(\boldsymbol{\mu}_{i}-\overline{\mathbf{x}}_{0}\right)\right] \\
\xi_{i} & =-\log \left(\frac{\left|\mathbf{H}_{0}\right|}{\left|\mathbf{H}_{1}\right|}\right)+\left(\boldsymbol{\mu}_{i}-\overline{\mathbf{x}}_{0}\right)^{T} \mathbf{H}_{0}\left(\boldsymbol{\mu}_{i}-\overline{\mathbf{x}}_{0}\right) \\
& -\left(\boldsymbol{\mu}_{i}-\overline{\mathbf{x}}_{1}\right)^{T} \mathbf{H}_{1}\left(\boldsymbol{\mu}_{i}-\overline{\mathbf{x}}_{1}\right)+2 \log \frac{\pi_{1}}{\pi_{0}}
\end{aligned}
$$

It entails from (2.5) that the misclassification error rate reduces to the cumulative distribution function (CDF) of bilinear forms of Gaussian random vectors, and as such cannot be derived in closed-form. However, as will be shown in the next section, an asymptotic evaluation of it can be obtained by utilizing the central limit theorem. 


\section{Chapter 3}

\section{Main Results}

In this part, we show that the classification error rate converges under some mild assumptions to a some deterministic quantity that depends on the means and covariances associated with each class. These assumptions are designed in such a way to avoid trivial misclassification error rates.

\subsection{Technical Assumptions}

The following assumptions are conceived in order to get non-trivial classification error rates. For $i \in\{0,1\}$, when $n_{i}, p \rightarrow \infty$, we make the following assumptions

Assumption 1 (Data scaling). $\frac{n_{i}}{p} \rightarrow c \in\{0, \infty\}$ with $\left|n_{0}-n_{1}\right|=o(1)$.

Assumption 2 (Mean scaling). Let $\boldsymbol{\mu} \triangleq \boldsymbol{\mu}_{0}-\boldsymbol{\mu}_{1},\|\boldsymbol{\mu}\|=\left\|\boldsymbol{\mu}_{0}-\boldsymbol{\mu}_{1}\right\|^{2}=\mathcal{O}(\sqrt{p})$.

Assumption 3 (Covariance scaling). $\left\|\boldsymbol{\Sigma}_{i}\right\|=\mathcal{O}(1)$.

Assumption 4 (Covariance separation). The matrix $\boldsymbol{\Sigma}_{0}-\boldsymbol{\Sigma}_{1}$ has exactly $\mathcal{O}(\sqrt{p})$ eigenvalues of $\mathcal{O}(1)$ while the remaining ones decay at an order of $\mathcal{O}(1 / \sqrt{p})$.

Assumption 1 establishes the double asymptotic regime which implies that the number of samples are commensurable with their dimensions. As a byproduct of Assumption 1, we have $\pi_{i} \rightarrow \frac{1}{2}$ as $n, p \rightarrow \infty$, and $\left|\alpha_{i}-\beta_{1-i}\right|=o(1)$. This particularly implies that the regularization weight associated with $\boldsymbol{\Sigma}_{i}$ in $\mathbf{H}_{0}$ is approximately equal to that corresponding to $\boldsymbol{\Sigma}_{1-i}$ in $\mathbf{H}_{1}$. This is useful from a technical perspective to control the distance between $\mathbf{H}_{1}$ and $\mathbf{H}_{0}$. Assumption 2 states that Euclidean distance 
of the difference between the means should scale at a rate of $\mathcal{O}(\sqrt{p})$ for RDA. As will be elaborated on later, this is the growth rate that allows RDA to leverage information about the means of both classes. Assumption 3 bounds the spectral norm of the covariance matrices and is of standard use in random matrix theory. Assumption 4 controls the distance between covariance matrices to avoid the situation of trivial misclassification error rates, which implies that $\frac{1}{\sqrt{p}} \operatorname{tr} \mathbf{A}\left(\Sigma_{0}-\Sigma_{1}\right)=\mathcal{O}(1)$ for any A with bounded spectral norm. Assumption 2 and Assumption 4 play important roles on controlling the distance between class means and class covariances so that the RDA classifier can present significative performance. The importance of these assumptions will be discussed later.

\subsection{Central Limit Theorem(CLT)}

Under Assumptions 1-4, using Lyapunov's CLT in [10], the bilinear form $\boldsymbol{\omega}^{T} \mathbf{B}_{i} \boldsymbol{\omega}+$ $2 \boldsymbol{\omega}^{T} \mathbf{y}_{i}$ in the random vector $\boldsymbol{\omega}$ with $\boldsymbol{\omega} \sim \mathcal{N}\left(\mathbf{0}, \mathbf{I}_{p}\right)$ can be proved to follow a Gaussian distribution with mean $\operatorname{tr} \mathbf{B}_{i}$ and variance $2 \operatorname{tr} \mathbf{B}_{i}^{2}+4 \mathbf{y}_{i}^{T} \mathbf{y}_{i}$. The detailed proof can be found in [11]. Based on this result, we thus prove that the condition classification error $\epsilon_{i}$ satisfies

Theorem 1. Assume $\lambda \neq 1$. Under assumptions 1 14 , the conditional classification error in 2.4 satisfies

$$
\epsilon_{i}^{R D A}-\Phi\left((-1)^{i} \frac{\xi_{i}-\operatorname{tr} \mathbf{B}_{i}}{\sqrt{2 \operatorname{tr} \mathbf{B}_{i}^{2}+4 \mathbf{y}_{i}^{T} \mathbf{y}_{i}}}\right) \stackrel{\text { a.s. }}{\longrightarrow} 0 .
$$

However, R-LDA, which has been studied in [7] could not be directly derived from Theorem 1, since it is associated with a linear classifier, while RDA involves in general a quadratic form in its classification rule. 


\subsection{Deterministic Equivalent}

In this part, we derive the asymptotic misclassification error rate. Prior to stating our results, we need to introduce the following notations which stems from the use of standard tools of random matrix theory. For $i \in\{0,1\}$, denote by $\delta_{i}$ the unique positive solution to the following fixed point equation

$$
\delta_{i}=\frac{1}{n_{i}} \operatorname{tr} \boldsymbol{\Sigma}_{i}\left[(1-\gamma) \mathbf{I}_{p}+\frac{\alpha_{i}}{1+\alpha_{i} \delta_{i}} \boldsymbol{\Sigma}_{0}+\frac{\beta_{i}}{1+\beta_{i} \delta_{i}} \boldsymbol{\Sigma}_{1}\right]^{-1}
$$

Define

$$
\begin{gathered}
\tilde{\delta}_{i}=\frac{\alpha_{i}}{1+\alpha_{i} \delta_{i}}, \\
\mathbf{Q}_{i}=\left[(1-\gamma) \mathbf{I}_{p}+\frac{\alpha_{i}}{1+\alpha_{i} \delta_{i}} \boldsymbol{\Sigma}_{0}+\frac{\beta_{i}}{1+\beta_{i} \delta_{i}} \boldsymbol{\Sigma}_{1}\right]^{-1}
\end{gathered}
$$

and for $j, k \in\{0,1\}$, let

$$
\phi_{i, j, k}=\frac{1}{n_{0}} \operatorname{tr} \Sigma_{i} \mathbf{Q}_{j} \Sigma_{i} \mathbf{Q}_{k}
$$

With these notations at hand, we prove the following convergences.

Proposition 1. Under Assumptions 1-4, we have

$$
\begin{gathered}
\frac{1}{\sqrt{p}} \xi_{i}-\bar{\xi}_{i} \stackrel{p}{\rightarrow} 0, \\
\frac{1}{\sqrt{p}} \operatorname{tr} \mathbf{B}_{i}-\bar{b}_{i} \stackrel{p}{\rightarrow} 0, \\
\frac{1}{p} \operatorname{tr} \mathbf{B}_{i}^{2}-\bar{B}_{i} \stackrel{p}{\rightarrow} 0,
\end{gathered}
$$


where

$$
\begin{aligned}
\bar{\xi}_{i} & \triangleq \frac{1}{\sqrt{p}} \log \left(\frac{1+\alpha_{0} \delta_{0}}{1+\alpha_{1} \delta_{1}}\right)^{n_{0}}\left(\frac{1+\beta_{0} \delta_{0}}{1+\beta_{1} \delta_{1}}\right)^{n_{1}}+\frac{1}{\sqrt{p}} \log \frac{\left|\mathbf{Q}_{1}\right|}{\left|\mathbf{Q}_{0}\right|} \\
& +\frac{1}{\sqrt{p}}\left[\frac{\alpha_{1} \delta_{1} n_{0}-\alpha_{0} \delta_{0} n_{0}}{\left(1+\alpha_{1} \delta_{1}\right)\left(1+\alpha_{0} \delta_{0}\right)}+\frac{\beta_{1} \delta_{1} n_{0}-\beta_{0} \delta_{0} n_{0}}{\left(1+\beta_{1} \delta_{1}\right)\left(1+\beta_{0} \delta_{0}\right)}\right] \\
& +\frac{1}{\sqrt{p}}(-1)^{i+1} \boldsymbol{\mu}^{T} \mathbf{Q}_{1-\mathbf{i}} \boldsymbol{\mu} . \\
\bar{b}_{i} & \triangleq \frac{1}{\sqrt{p}} \operatorname{tr} \boldsymbol{\Sigma}_{i}\left(\mathbf{Q}_{1}-\mathbf{Q}_{0}\right) . \\
\bar{B}_{i} & \triangleq \frac{1}{p} \frac{\phi_{i, 0,0}}{1-\left(\frac{\tilde{\delta}_{1}^{2}}{n_{1}}+\frac{\tilde{\delta}_{0}^{2}}{n_{0}}\right) \phi_{i, 0,0}}+\frac{1}{p} \frac{\phi_{i, 1,1}}{1-\left(\frac{\tilde{\delta}_{1}^{2}}{n_{1}}+\frac{\tilde{\delta}_{1}^{2}}{n_{0}}\right) \phi_{i, 1,1}}-\frac{2}{p} \frac{\phi_{i, 1,0}}{1-2 \frac{\tilde{\delta}_{1} \tilde{\delta}_{0}}{n_{0}} \phi_{i, 1,0}} .
\end{aligned}
$$

The detailed proofs are provided in Appendix A. Plugging these deterministic equivalents into the misclassification error rate in Theorem 1, we obtain the following theorem.

Theorem 2. Under assumptions 1 近, the following convergence holds for $i \in\{0,1\}$

$$
\epsilon_{i}^{R D A}-\Phi\left((-1)^{i} \frac{\bar{\xi}_{i}-\overline{b_{i}}}{\sqrt{2 \bar{B}_{i}}}\right) \stackrel{p}{\rightarrow} 0
$$

Proof. It appears that $\bar{\xi}_{i}$ and $\bar{b}_{i}$ are going to blow up at the rate of $\mathcal{O}(\sqrt{p})$ so that the classification error will converge to a trivial value since $\frac{1}{\sqrt{p}} \log \left|\mathbf{Q}_{i}\right|$ and $\frac{1}{\sqrt{p}} \operatorname{tr} \Sigma_{i} Q_{i}$ are $\mathcal{O}(\sqrt{p})$. However, because of Assumption 4, the distance of class covariances are controlled, which results in $\frac{1}{\sqrt{p}} \log \left|\mathbf{Q}_{1}\right|-\frac{1}{\sqrt{p}} \log \left|\mathbf{Q}_{0}\right|$ being $\mathcal{O}(1)$ and $\frac{1}{\sqrt{p}} \operatorname{tr} \boldsymbol{\Sigma}_{i}\left(\mathbf{Q}_{1}-\mathbf{Q}_{0}\right)$ being $\mathcal{O}(1)$. Take $\frac{1}{\sqrt{p}} \operatorname{tr} \boldsymbol{\Sigma}_{i}\left(\mathbf{Q}_{1}-\mathbf{Q}_{0}\right)$ for example. We first use resolvent identity 
$\mathbf{A}^{-1}-\mathbf{B}^{-1}=\mathbf{A}^{-1}(\mathbf{B}-\mathbf{A}) \mathbf{B}^{-1}$. to expand $\bar{b}_{i}$ as follows:

$$
\begin{aligned}
\bar{b}_{i} & =\frac{1}{\sqrt{p}} \operatorname{tr} \boldsymbol{\Sigma}_{i}\left(\mathbf{Q}_{1}-\mathbf{Q}_{0}\right) \\
& =\frac{1}{\sqrt{p}} \operatorname{tr} \boldsymbol{\Sigma}_{i} \mathbf{Q}_{1}\left(\mathbf{Q}_{0}^{-1}-\mathbf{Q}_{1}^{-1}\right) \mathbf{Q}_{0} \\
& =\frac{1}{\sqrt{p}} \operatorname{tr} \boldsymbol{\Sigma}_{i} \mathbf{Q}_{1}\left(\frac{\alpha_{0}}{1+\alpha_{0} \delta_{0}} \boldsymbol{\Sigma}_{0}+\frac{\beta_{0}}{1+\beta_{0} \delta_{0}} \boldsymbol{\Sigma}_{1}-\frac{\alpha_{1}}{1+\alpha 1 \delta_{1}} \boldsymbol{\Sigma}_{0}-\frac{\beta_{1}}{1+\beta_{1} \delta_{1}} \boldsymbol{\Sigma}_{1}\right) \mathbf{Q}_{0} \\
& =\frac{1}{\sqrt{p}}\left(\frac{\alpha_{0}}{1+\alpha_{0} \delta_{0}}-\frac{\alpha_{1}}{1+\alpha_{1} \delta_{1}}\right) \operatorname{tr} \mathbf{Q}_{0} \boldsymbol{\Sigma}_{i} \mathbf{Q}_{1}\left(\boldsymbol{\Sigma}_{0}-\boldsymbol{\Sigma}_{1}\right)+\mathcal{O}\left(\frac{1}{\sqrt{p}}\right)
\end{aligned}
$$

Since $\left\|Q_{0} \Sigma_{i} Q_{1}\right\|$ is bounded, from Assumption 4, we can derive that $\frac{1}{\sqrt{p}}\left(\frac{\alpha_{0}}{1+\alpha_{0} \delta_{0}}-\right.$ $\left.\frac{\alpha_{1}}{1+\alpha_{1} \delta_{1}}\right) \operatorname{tr} \mathbf{Q}_{0} \Sigma_{i} \mathbf{Q}_{1}\left(\Sigma_{0}-\Sigma_{1}\right)=\mathcal{O}(1)$. Therefore, we complete the convergence proof for $\bar{b}_{i}$ under Assumption 4. It is also easy to prove that $\frac{1}{\sqrt{p}}(-1)^{i+1} \boldsymbol{\mu}^{T} \mathbf{Q}_{\mathbf{1}-\mathbf{i}} \boldsymbol{\mu}=\mathcal{O}(1)$ using Assumption 2. In conclusion, these assumptions are carefully established to guarantee a non-trivial classification error.

Theorem 2 reveals two important facts. First, the classification error rate can be characterized asymptotically by a deterministic quantity that depends solely on the parameters of the Gaussian model as well as the problem dimensions. Second, the importance of this result lies in that it not only sheds light on the impact of these parameters but also it opens up possibilities of properly tuning the pair of $\gamma$ and $\lambda$ that correspond to the least asymptotic misclassification error rate.

Remark 1. Under Assumption 4, it is easy to show that $\phi_{i, 0,0}, \phi_{i, 1,1}$ and $\phi_{i, 1,0}$ are asymptotically equivalent, therefore $\overline{B_{i}}$ can be further simplified to

$$
\overline{B_{i}} \triangleq \frac{1}{p} \frac{2 n_{1} \phi}{1-\left(\tilde{\delta}_{1}^{2}+\tilde{\delta}_{0}^{2}\right) \phi}-\frac{1}{p} \frac{2 n_{1} \phi}{1-2 \tilde{\delta}_{0} \tilde{\delta}_{1} \phi}+o(1)
$$

where $\phi$ can be any $\phi_{i, j, k}$. This simplification is due to the fact that the interchangeable use of $\boldsymbol{\Sigma}_{0}$ and $\boldsymbol{\Sigma}_{1}$ will only cause an error of order o(1). Under this condition, we can treat $\overline{B_{0}}$ and $\overline{B_{1}}$ as equivalent in the asymptotic sense. However, in the simulation 
we prefer to use the original expression to guarantee a result with higher accuracy.

Special cases:

1. As shown in [8], for the R-LDA to leverage the information about the mean classes, it suffices to have $\left\|\boldsymbol{\mu}_{0}-\boldsymbol{\mu}_{1}\right\|=\mathcal{O}$ (1). In our case, when $\left\|\boldsymbol{\mu}_{0}-\boldsymbol{\mu}_{1}\right\|=$ $\mathcal{O}(1)$, the classification error of RDA can still converge to a non-trivial deterministic equivalence in which the contribution of the difference in means vanishes. This is because RDA suffers from a higher level of estimation noise, requiring the distance between the means $\left\|\boldsymbol{\mu}_{0}-\boldsymbol{\mu}_{1}\right\|$ to scale as high as $O(\sqrt{p})$ so as to be leveraged by the classifier.

2. When $\left\|\boldsymbol{\Sigma}_{0}-\boldsymbol{\Sigma}_{1}\right\|_{F}=\mathcal{O}(1)$ and $\left\|\boldsymbol{\mu}_{0}-\boldsymbol{\mu}_{1}\right\|=\mathcal{O}(1)$, it is easy to show that $\bar{b}_{0}-$ $\bar{b}_{1} \rightarrow 0$. Therefore, we can show that $\epsilon_{0}^{R D A}=\phi(\omega)$ and $\epsilon_{1}^{R D A}=\phi(-\omega)$ where $\omega$ is some quantity that is the same for both classes. Finally, the classification error $\epsilon$ will converge to $\pi_{0} \epsilon_{0}^{R D A}+\pi_{1} \epsilon_{1}^{R D A}=0.5 \phi(\omega)+0.5 \phi(-\omega)=0.5$. This indicates that when $\left\|\Sigma_{0}-\Sigma_{1}\right\|_{F}=O(1)$, the information about the covariance matrices is not exploited. In such circumstances, the use of R-LDA should be firstly considered. 


\subsection{Validation with Synthetic Data}

In this chapter, we provide numerical results to validate the accuracy of our theoretical findings. Define the following set of parameters for the Gaussian model: $\left[\boldsymbol{\Sigma}_{0}\right]_{i, j}=0.6^{|i-j|}, \boldsymbol{\Sigma}_{1}=\boldsymbol{\Sigma}_{0}+2\left[\begin{array}{cc}\mathbf{I}_{k} & \mathbf{O}_{k \times(p-k)} \\ \mathbf{O}_{(p-k) \times k} & \mathbf{O}_{(p-k) \times(p-k)}\end{array}\right]$ and $k=\lfloor\sqrt{p}\rfloor$. The statistical means are taken to be $\boldsymbol{\mu}_{0}=\mathbf{1}_{p \times 1}$ and $\boldsymbol{\mu}_{1}=\boldsymbol{\mu}_{0}+2 p^{-\frac{1}{4}} \mathbf{1}_{p \times 1}$. In the first experiment, we validate the correctness of our derived asymptotic error. We first estimate the means and covariances from the training data set and compute the empirical error by drawing $n_{\text {test }}=2000$ samples from testing data set. We run 200 Monte carlo simulations for this process and take the average value for the final empirical error. Fixing $\gamma=0.5, \lambda=0.5$ and $p$ varying from 100 to 500, Figure 3.1 shows the behavior of the classification error rate. We can see that the asymptotic error presents good agreement with the empirical error computed over the testing data. In a second experiment, taking $p \in\{300,400,500\}$ and $c=1$, we want to verify what trends the classification error will present with the variation of both regularization parameters $\gamma$ and $\lambda$. Since our derivations for RDA cannot generalize to R-LDA, we will compare the performance of R-LDA with RDA in terms of classification error separately. Finally, we get the 3D plots about RDA and R-LDA's variation trends of classification errors as shown in Figure 3.2. The blue curved planes are the misclassification error of R-LDA with variation of $\gamma$ and the colorful surfaces are misclassification error of RDA with variation of $\gamma$ and $\lambda$. It is clear from the plots that the minimum classification error is achieved in the most red region of the RDA surface below the R-LDA curved plane. Regularization parameter $\lambda$ approaching the minimum classification error is neither 1 nor 0 , which means that the optimal classifier minimizing the classification error is not one of the extreme cases, namely, neither $\operatorname{R-LDA}(\lambda=1)$ nor R-QDA $(\lambda=0)$. Instead, it lies somewhere between R-LDA and R-QDA, which aligns with our expectation that RDA offers better classification performance than R-LDA and 


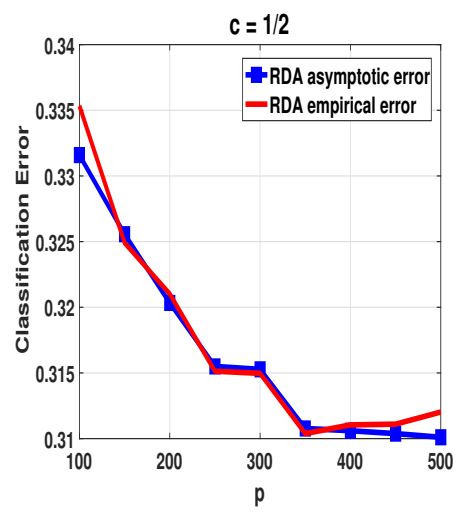

(a)

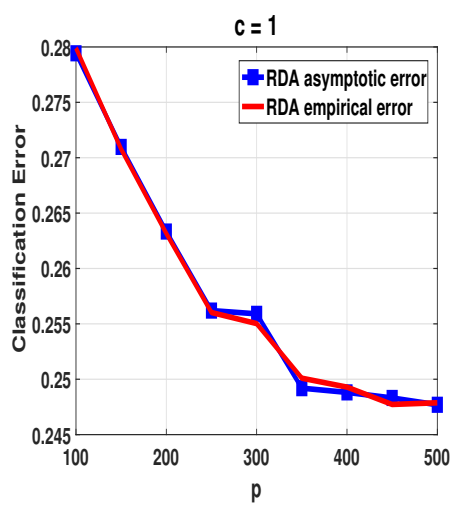

(b)

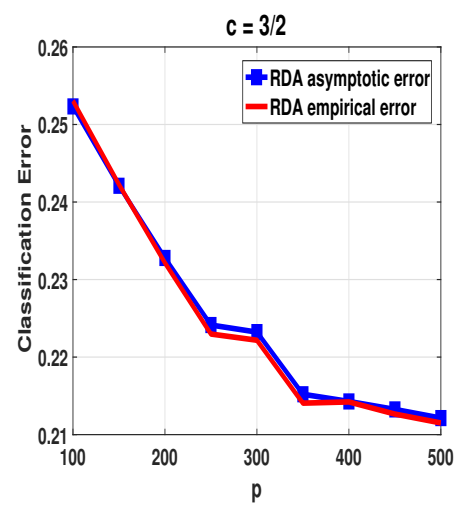

(c)

Figure 3.1: RDA classifier performance in terms of classification error with equal training, $n_{0}=n_{1}$. The $x$ axis is the number of the data dimension.

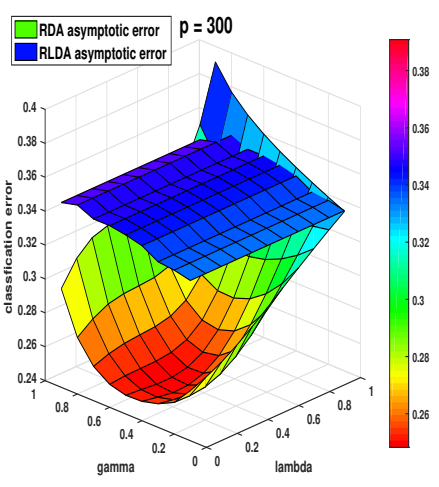

(a)

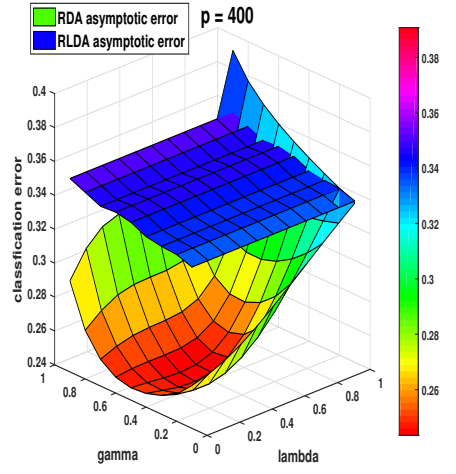

(b)

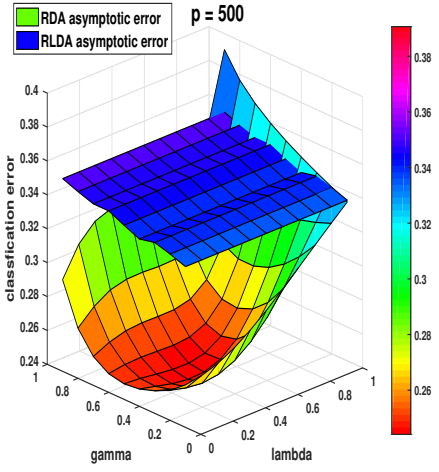

(c)

Figure 3.2: Comparison of performance of RDA and R-LDA in terms of classification error with equal training, $n_{0}=n_{1}$. The $x$ axis is regularization parameter $\lambda$ and $y$ axis is regularization parameter $\gamma$ for $p \in\{300,400,500\}$ and $c=1$.

R-QDA with proper regularizers selection. 


\section{Chapter 4}

\section{General Consistent Estimator for RDA}

\subsection{General Consistent Estimator of the Testing Error}

The theoretical results we derived in previous chapter serve to provide a better understanding about the relationship between the classification error and the statistics for us. However, in practice, it is usually unavailable to know the class covariances and means in advance. In this chapter, we resolve this issue by using the estimated class statistics from the training data to establish the general consistent estimator. The general estimator is constructed to precisely approximate the behavior of the deterministic one in previous chapter. There are also some other widely used estimators such as cross validation based ones which can produce estimated classification in close proximity to the true error. We employ both synthetic and real data to compare the performance of our proposed estimator with other well-known estimator. It turns out that the general consistent estimator wins in terms of measure metric such as MSE. Based on the results of deterministic equivalent of the conditional classification er-

ror we derived in Theorem 2, we construct a general consistent estimator of $\epsilon_{i}^{R D A}$ denoted by $\hat{\epsilon}_{i}^{R D A}$. Before providing the expression of $\hat{\epsilon}_{i}^{R D A}$, we shall introduce some useful quantities.

$$
\begin{gathered}
\eta_{i}=\frac{1}{n_{i}} \operatorname{tr} \boldsymbol{\Sigma}_{i} \mathbf{H}_{1}=\frac{1}{n_{i}} \frac{\operatorname{tr} \widehat{\Sigma}_{i} \mathbf{H}_{1}}{1-\frac{\beta_{i}}{n_{i}} \operatorname{tr} \widehat{\boldsymbol{\Sigma}}_{i} \mathbf{H}_{1}} \\
\tau_{i}=\frac{1}{n_{i}} \operatorname{tr} \boldsymbol{\Sigma}_{i} \mathbf{H}_{0}=\frac{1}{n_{i}} \frac{\operatorname{tr} \widehat{\boldsymbol{\Sigma}}_{i} \mathbf{H}_{0}}{1-\frac{\beta_{1-i}}{n_{i}} \operatorname{tr} \widehat{\boldsymbol{\Sigma}}_{i} \mathbf{H}_{0}}
\end{gathered}
$$


Now we are in position to give the theorem approximating the RDA misclassification error by general consistent estimators.

Theorem 3. Under Assumptions 1, 4, define

$$
\widehat{\epsilon}_{i}^{R D A}=\Phi\left((-1)^{i} \frac{\widehat{\xi}_{i}-\widehat{b}_{i}}{\sqrt{2 \widehat{B}_{i}}}\right)
$$

Then,

$$
\widehat{\epsilon}_{i}^{R D A}-\epsilon_{i}^{R D A} \stackrel{p}{\rightarrow} 0
$$

where

$$
\begin{aligned}
\widehat{\xi}_{i} & =-\frac{1}{\sqrt{p}} \log \frac{\left|\mathbf{H}_{0}\right|}{\left|\mathbf{H}_{1}\right|}+\frac{(-1)^{i+1}}{\sqrt{p}}\left(\overline{\mathbf{x}}_{0}-\overline{\mathbf{x}}_{1}\right)^{T} \mathbf{H}_{1-i}\left(\overline{\mathbf{x}}_{0}-\overline{\mathbf{x}}_{1}\right) . \\
\widehat{b}_{i} & =\frac{n_{i}}{\sqrt{p}}\left(\eta_{i}-\tau_{i}\right) . \\
\widehat{B}_{i} & =\left(1+\beta_{i} \eta_{i}\right)^{4} \frac{1}{p} \operatorname{tr} \widehat{\boldsymbol{\Sigma}}_{i} \mathbf{H}_{1} \widehat{\boldsymbol{\Sigma}}_{i} \mathbf{H}_{1}-\eta_{i}^{2}\left(1+\beta_{i} \eta_{i}\right)^{2}+\left(1+\beta_{1-i} \tau_{i}\right)^{4} \frac{1}{p} \operatorname{tr} \widehat{\boldsymbol{\Sigma}}_{i} \mathbf{H}_{0} \widehat{\boldsymbol{\Sigma}}_{i} \mathbf{H}_{0} \\
& -\tau_{i}^{2}\left(1+\beta_{1-i} \tau_{i}\right)^{2}-2\left(1+\beta_{i} \eta_{i}\right)^{2}\left(1+\beta_{1-i} \tau_{i}\right)^{2} \frac{1}{p} \operatorname{tr} \widehat{\boldsymbol{\Sigma}}_{i} \mathbf{H}_{1} \widehat{\boldsymbol{\Sigma}}_{i} \mathbf{H}_{0} \\
& +2 \eta_{i}^{2} \tau_{i}^{2}\left(1+\beta_{i} \eta_{i}\right)\left(1+\beta_{1-i} \tau_{i}\right) .
\end{aligned}
$$

The proof is provided in Appendix B.

\subsection{Validation with Synthetic Data}

In this chapter, we provide numerical results to validate the accuracy of our theoretical findings. Define the following set of parameters for the Gaussian model: $\left[\boldsymbol{\Sigma}_{0}\right]_{i, j}=0.6^{|i-j|}, \boldsymbol{\Sigma}_{1}=\boldsymbol{\Sigma}_{0}+4\left[\begin{array}{cc}\mathbf{I}_{k} & \mathbf{0}_{k \times(p-k)} \\ \mathbf{0}_{(p-k) \times k} & \mathbf{0}_{(p-k) \times(p-k)}\end{array}\right]$. The statistical means are taken to be $\boldsymbol{\mu}_{0}=\mathbf{1}_{p \times 1}$ and $\boldsymbol{\mu}_{1}=\boldsymbol{\mu}_{0}+2 p^{-\frac{1}{4}} \mathbf{1}_{p \times 1}$. In the first experiment, we validate 
the correctness of our derived general consistent estimated misclassification error. We first estimate the means and covariances from the training data set and compute the empirical error by drawing $n_{\text {test }}=2000$ samples from testing data set. We run 200 monte-carlo simulations for this process and take the average value for the final empirical error. Fixing $\gamma=0.5, \lambda=0.5$ and $p$ varying from 100 to 1500 , Figure 4.1 shows the behavior of the classification error rate. We can see that the error rate of general consistent estimator presents good agreement with the empirical error computed over the testing data. In the second experiment, we compare the performance of our proposed general consistent estimator with other estimators including cross validation estimator and plug in estimator in terms of MSE. From Figure 4.2, we can see that the plug in estimator presents the worst approximation. This is due to the fact that when both the data dimension and training size increases in the same pace, the estimated class covariance and mean cannot closely approximate the true class statistics. The results also show that our general estimator always achieves better MSE performance than the cross validation estimator. 


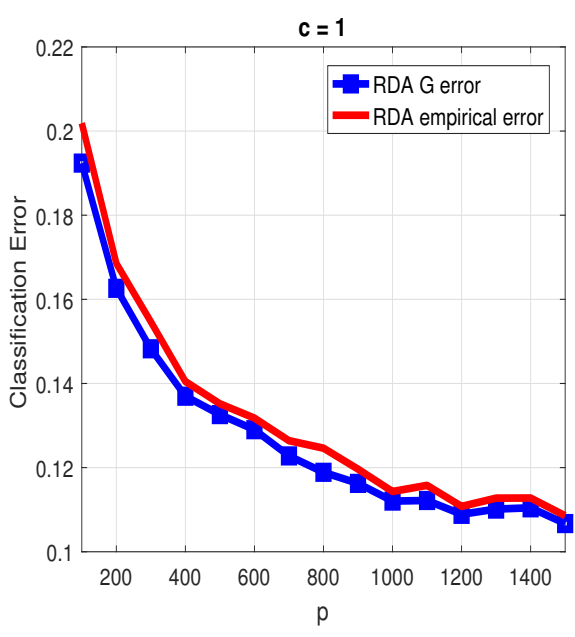

(a)

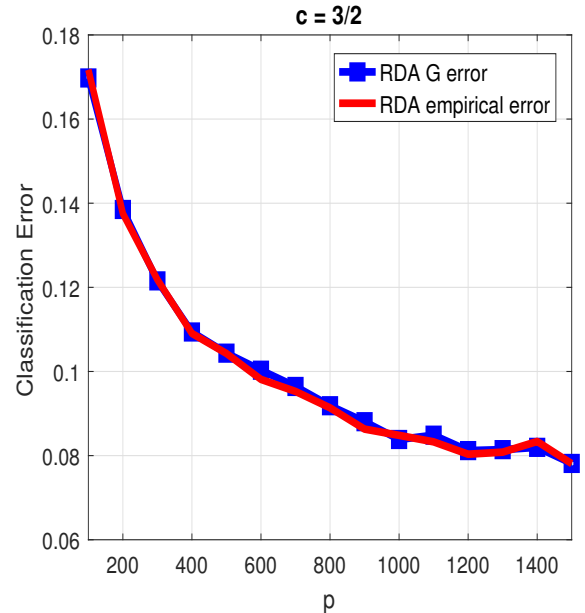

(b)

Figure 4.1: RDA general consistent estimator performance in terms of classification error with equal training, $n_{0}=n_{1}$. The $x$ axis is the number of the data dimension.

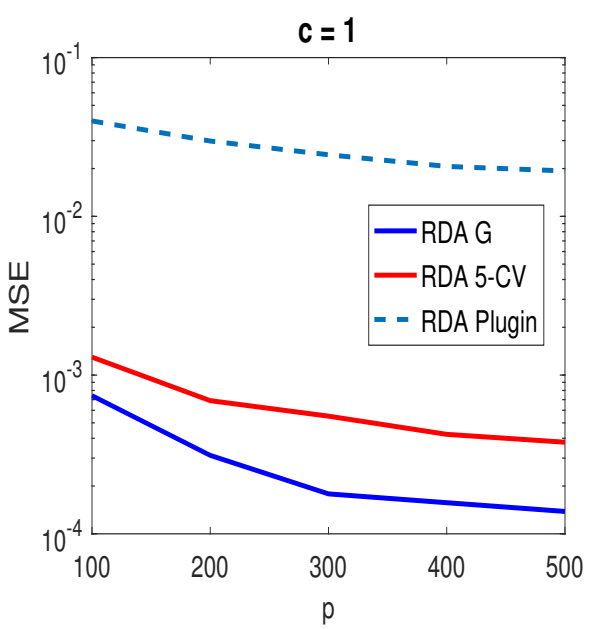

(a)

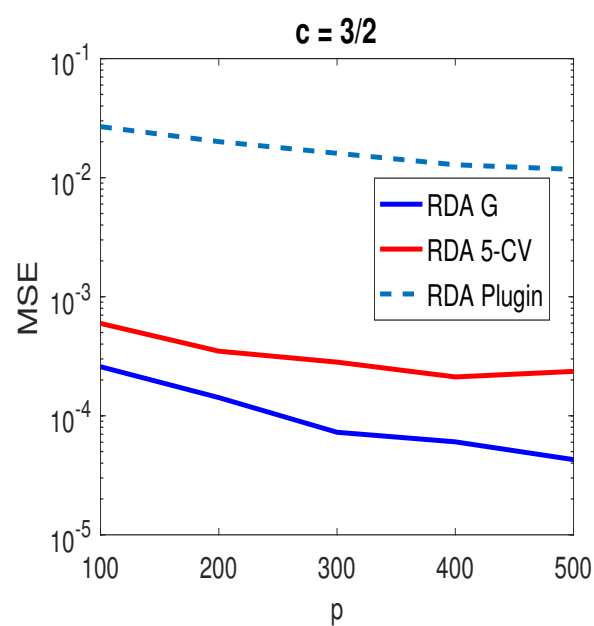

(b)

Figure 4.2: MSE performance of the proposed general consistent estimators compared with 5 -fold cross validation and plug in estimator.

\subsection{Validation with Real Data}

In this section, we use the real USPS dataset (http://www.csie.ntu.edu.tw/ cjlin/ libsvmtools/datasets/multiclass.html\#usps) to compare the performance of our estimator with the cross validation estimator. The dataset consists of 7291 training samples of $16 \times 16$ grayscale images ( $p=256$ features) and 2007 testing images. We 


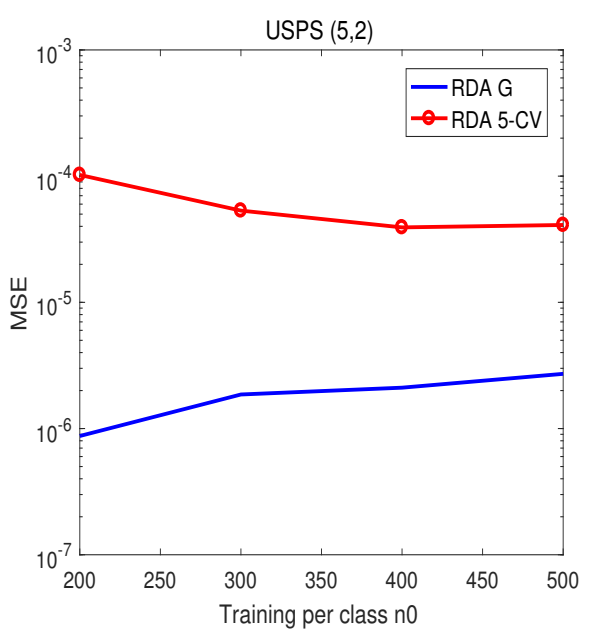

(a)

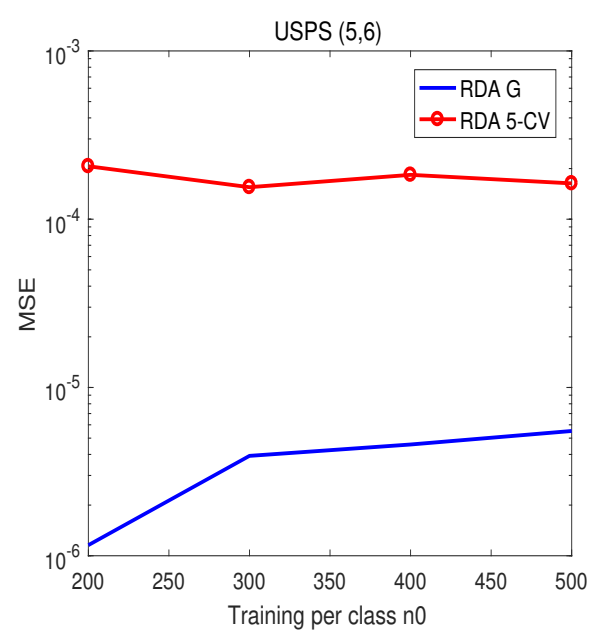

(b)

Figure 4.3: MSE performance of the proposed general consistent estimators compared with 5 -fold cross validation and plug in estimator. We choose equal training $n_{0}=n_{1}$.

examine the MSE performance of the different error estimators on the data with variation of training size. We choose 2 group of class labels digit $(5,2)$ and $(5,6)$ to do the experiments. Similar to the synthetic data case, our general estimator outperforms the 5 fold cross validation again. 


\section{Chapter 5}

\section{Concluding Remarks}

\section{$5.1 \quad$ Summary}

In this thesis, we consider the double asymptotic regime where the sample size and data dimension increase comparatively in magnitude to study the performance of the RDA classifier. Under some mild assumptions controlling the distance between the class means and covariances, we show that the asymptotic classification error converges to a deterministic quantity relying merely on the data dimension and statistics of each class. This conclusion enables us to design an improved classifier by selecting a pair of regularization parameters that minimize the asymptotic classification error. We validate the accuracy of our theoretical findings using synthetic data which allows to see the advantage of using the RDA classifier as compared to its special cases R-LDA and R-QDA. Our designed general consistent estimator also shows a great approximation to the real classification error and outperforms other traditional estimators both on the synthetic data set and real data set.

\subsection{Future Research Work}

The future work could be extended to study the some unsupervised algorithms for clustering like k-means. Or we can consider other statistical learning techniques where there is a room for random matrix theory. For example, we can study kernel machines, neural networks. 


\section{REFERENCES}

[1] G. McLachlan, Discriminant analysis and statistical pattern recognition. John Wiley \& Sons, 2004, vol. 544.

[2] D. Bakirov, A. P. James, and A. Zollanvari, "An efficient method to estimate the optimum regularization parameter in RLDA," Bioinformatics, vol. 32, no. 22, pp. 3461-3468, 2016.

[3] P. Ou, "Prediction of Stock Market Index Movement by Ten Data Mining Techniques," Modern Applied Science, vol. 3, no. 12, Dec. 2009.

[4] J. Friedman, "Regularized discriminant analysis," Journal of the American Statistical Association, vol. 84, pp. 165-175, 1989.

[5] H. R. McFarland and D. S. P. Richards, "Exact misclassification probabilities for plug-in normal quadratic discriminant functions: Ii. the heterogeneous case," Journal of Multivariate Analysis, vol. 82, no. 2, pp. 299-330, 2002.

[6] E. P. Wigner, "On the distribution of the roots of certain symmetric matrices," Annals of Mathematics, pp. 325-327, 1958.

[7] A. Zollanvari and E. R. Dougherty, "Generalized consistent error estimator of linear discriminant analysis," IEEE transactions on signal processing, vol. 63, no. 11, pp. 2804-2814, 2015.

[8] K. Elkhalil, A. Kammoun, R. Couillet, T. Y. Al-Naffouri, and M. S. Alouini, "Asymptotic performance of regularized quadratic discriminant analysis based classifiers," in IEEE 27th International Workshop on Machine Learning for Signal Processing (MLSP), Sept 2017, pp. 1-6.

[9] J. Friedman, T. Hastie, and R. Tibshirani, The elements of statistical learning. Springer series in statistics New York, 2001, vol. 1.

[10] P. Billingsley, Probability and measure. John Wiley \& Sons, 2008.

[11] K. Elkhalil, A. Kammoun, R. Couillet, T. Y. Al-Naffouri, and M.-S. Alouini, "A Large Dimensional Study of Regularized Discriminant Analysis Classifiers," ArXiv e-prints, Nov. 2017. 
[12] F. Benaych-Georges and R. Couillet, "Spectral analysis of the gram matrix of mixture models," ESAIM: Probability and Statistics, vol. 20, pp. 217-237, 2016.

[13] R. Couillet, M. Debbah, and J. W. Silverstein, "A deterministic equivalent for the analysis of correlated mimo multiple access channels," IEEE Transactions on Information Theory, vol. 57, no. 6, pp. 3493-3514, 2011.

[14] R. Couillet and M. Debbah, Random matrix methods for wireless communications. Cambridge University Press, 2011.

[15] W. H. Press, Numerical recipes 3rd edition: The art of scientific computing. Cambridge university press, 2007. 


\section{APPENDICES}




\section{A Proof of Proposition 1}

First, we introduce the definitions that will be used in the following proofs. For $i \in$ $\{0,1\}$, define $\mathbf{Y}_{i}=\Sigma_{i}^{1 / 2} \mathbf{Z}_{i}$ and $\mathbf{Z}_{i}=\left[\mathbf{z}_{i, 1}, \cdots, \mathbf{z}_{i, n_{i}}\right] \in \mathbb{R}^{p \times n_{i}}$ such that $\mathbf{z}_{i, j}$ are random vectors with standard multivariate Gaussian distribution. By simple transformation, we can write $\mathbf{H}_{i}$ as

$$
\mathbf{H}_{i}=\left(\alpha_{i} \frac{\mathbf{Y}_{0} \mathbf{Y}_{0}^{T}}{n_{0}}+\beta_{i} \frac{\mathbf{Y}_{1} \mathbf{Y}_{1}^{T}}{n_{1}}+(1-\gamma) \mathbf{I}_{p}\right)^{-1}
$$

$\mathbf{H}_{i}$ is not a deterministic quantity since it involves random quantities. Therefore, we will first find its deterministic equivalence by applying the results presented in Benaych-Georges and Couillet[12.

$$
\widetilde{\mathbf{Q}}=\left(\frac{\mathbf{Y}_{0} \mathbf{Y}_{0}^{T}}{p}+\frac{\mathbf{Y}_{1} \mathbf{Y}_{1}^{T}}{p}-z \mathbf{I}_{p}\right)^{-1}
$$

$\overline{\widetilde{\mathbf{Q}}}$ is the deterministic equivalence of $\widetilde{\mathbf{Q}}$ :

$$
\overline{\widetilde{\mathbf{Q}}}(z) \triangleq-\frac{1}{z}\left(\mathbf{I}_{p}+c_{0} g_{0}(z) \boldsymbol{\Sigma}_{0}+c_{1} g_{1}(z) \boldsymbol{\Sigma}_{1}\right)^{-1}, z \in \mathbb{C} .
$$

where $g_{i}(z), i \in\{0,1\}$, satisfies the following fixed point equations

$$
\frac{p}{n} g_{i}(z)=-\frac{1}{z} \frac{1}{1+\tilde{g}_{i}(z)}, \quad \tilde{g}_{i}(z)=\frac{1}{p} \operatorname{tr} \Sigma_{i} \overline{\widetilde{\mathbf{Q}}}(z) .
$$


By normalizing $\widehat{\mathbf{Y}}_{0}=\sqrt{\frac{p \alpha_{i}}{n_{0}}} \mathbf{Y}_{0}$ and $\widehat{\mathbf{Y}}_{1}=\sqrt{\frac{p \beta_{i}}{n_{1}}} \mathbf{Y}_{1}$, we can rewrite $\mathbf{H}_{i}$ as

$$
\mathbf{H}_{i}=\left(\frac{\widehat{\mathbf{Y}}_{0} \widehat{\mathbf{Y}}_{0}^{T}}{p}+\frac{\widehat{\mathbf{Y}}_{1} \widehat{\mathbf{Y}}_{1}^{T}}{p}+(1-\gamma) \mathbf{I}_{p}\right)^{-1}
$$

and by choosing $z=\gamma-1$, we can model $\mathbf{H}_{i}$ as $\widetilde{\mathbf{Q}}_{i}$. Denote the deterministic equivalence of $\mathbf{H}_{i}$ as $\mathbf{Q}_{i}$. $\mathbf{Q}_{i}$ can be derived by using A.3 and A.4. Furthermore, we have the following equivalence.

$$
\begin{aligned}
& \frac{1}{p} \operatorname{tr} \mathbf{A}(\overline{\widetilde{\mathbf{Q}}}(z)-\widetilde{\mathbf{Q}}(z)) \rightarrow 0 . \\
& \mathbf{u}^{T}(\overline{\widetilde{\mathbf{Q}}}(z)-\widetilde{\mathbf{Q}}(z)) \mathbf{v} \rightarrow 0
\end{aligned}
$$

for all deterministic matrices $\mathbf{A}$ of bounded spectral norms and all deterministic vectors $\mathbf{u}$ and $\mathbf{v}$ of bounded euclidean norms. Applying this equivalent relation, we can easily prove $\frac{1}{\sqrt{p}} \operatorname{tr} \mathbf{B}_{i}-\overline{b_{i}} \stackrel{p}{\rightarrow} 0$. The proof consists in showing the following convergences which we prove below

$$
\begin{aligned}
& \frac{1}{\sqrt{p}} \xi_{i}-\bar{\xi}_{i} \stackrel{p}{\rightarrow} 0 . \\
& \frac{1}{\sqrt{p}} \operatorname{tr} \mathbf{B}_{i}-\overline{b_{i}} \stackrel{\text { a.s. }}{\longrightarrow} 0 . \\
& \frac{1}{p} \operatorname{tr} \mathbf{B}_{i}^{2}-\overline{B_{i}} \stackrel{\text { a.s. }}{\longrightarrow} 0 . \\
& \frac{1}{p} \mathbf{y}_{i}^{T} \mathbf{y}_{i} \stackrel{\text { a.s. }}{\longrightarrow} 0 .
\end{aligned}
$$

\section{A.0.1 Proof of A.7}

For the proof of $\overline{\xi_{i}}$, we will divide it into two parts to show the convergence of each one: the log-term and the remaining term.

$$
\frac{1}{\sqrt{p}} \xi_{i}=\frac{1}{\sqrt{p}}\left[-\log \left(\frac{\left|\mathbf{H}_{0}\right|}{\left|\mathbf{H}_{1}\right|}\right)+\left(\boldsymbol{\mu}_{i}-\overline{\mathbf{x}}_{0}\right)^{T} \mathbf{H}_{0}\left(\boldsymbol{\mu}_{i}-\overline{\mathbf{x}}_{0}\right)-\left(\boldsymbol{\mu}_{i}-\overline{\mathbf{x}}_{1}\right)^{T} \mathbf{H}_{1}\left(\boldsymbol{\mu}_{i}-\overline{\mathbf{x}}_{1}\right)+2 \log \frac{\pi_{1}}{\pi_{0}}\right]
$$


We first derive the deterministic equivalence for $-\frac{1}{\sqrt{p}} \log \left(\frac{\left|\mathbf{H}_{0}\right|}{\left|\mathbf{H}_{1}\right|}\right)$ using the model in [13]. To start with, we write

$$
\frac{1}{\sqrt{p}} \log \left(\frac{\left|\mathbf{H}_{0}\right|}{\left|\mathbf{H}_{1}\right|}\right)=\frac{1}{\sqrt{p}}\left(-\log \left|\mathbf{H}_{0}\right|+\log \left|\mathbf{H}_{1}\right|\right)
$$

Define $V_{i}$ as follows:

$$
\begin{aligned}
V_{i} & =\frac{1}{p} \log \left|\mathbf{I}_{p}+\frac{1}{1-\gamma}\left(\alpha_{i} \widehat{\boldsymbol{\Sigma}}_{0}+\beta_{i} \widehat{\boldsymbol{\Sigma}}_{1}\right)\right| \\
& =\frac{1}{p} \log \left|(1-\gamma) \mathbf{I}_{p}+\alpha_{i} \widehat{\boldsymbol{\Sigma}}_{0}+\beta_{i} \widehat{\boldsymbol{\Sigma}}_{1}\right|-\log (1-\gamma) \\
& =-\frac{1}{p} \log \left|\mathbf{H}_{\mathbf{i}}\right|-\log (1-\gamma)
\end{aligned}
$$

Thus,

$$
-\frac{1}{p} \log \left|\mathbf{H}_{\mathbf{i}}\right|=V_{i}+\log (1-\gamma)
$$

From corollary 1 in [13, we can derive the following deterministic equivalence for $V_{i}$.

$$
\widetilde{V}_{i} \rightarrow V_{i}
$$

According to the results from corollary 1 in [13], $e_{i 0}, m_{i 0}, e_{i 1}$ and $m_{i 1}$ are the solutions to the following equations.

$$
\begin{gathered}
\mathbf{T}_{i}=\mathbf{I}_{p}+m_{i 0} \alpha_{i} \boldsymbol{\Sigma}_{0}+m_{i 1} \beta_{i} \boldsymbol{\Sigma}_{1} \\
e_{i 0}=\frac{1}{p(1-\gamma)} \operatorname{tr}\left(\alpha_{i} \boldsymbol{\Sigma}_{0} \mathbf{T}_{i}^{-1}\right) \\
m_{i 0}=\frac{1}{(1-\gamma)\left(1+\frac{p e_{i 0}}{n_{0}}\right)} \\
e_{i 1}=\frac{1}{p(1-\gamma)} \operatorname{tr}\left(\beta_{i} \boldsymbol{\Sigma}_{1} \mathbf{T}_{i}^{-1}\right)
\end{gathered}
$$




$$
\begin{gathered}
m_{i 1}=\frac{38}{(1-\gamma)\left(1+\frac{p e_{i 1}}{n_{1}}\right)} \\
\widetilde{V}_{i}=\frac{1}{p} \log \left[\left(1+\frac{p e_{i 0}}{n_{0}}\right)^{n_{0}}\left(1+\frac{p e_{i 1}}{n_{1}}\right)^{n_{1}}\right]+\frac{1}{p} \log \left|\mathbf{T}_{\mathbf{i}}\right|-(1-\gamma)\left(m_{i 0} e_{i 0}+m_{i 1} e_{i 1}\right)
\end{gathered}
$$

Therefore, the deterministic equivalence for $-\frac{1}{p} \log \left|\mathbf{H}_{\mathbf{i}}\right|$ is

$$
-\frac{1}{p} \log \left|\mathbf{H}_{\mathbf{i}}\right| \rightarrow \widetilde{V}_{i}+\log (1-\gamma)
$$

Finally, we derive the following equivalence

$$
\begin{aligned}
-\frac{1}{\sqrt{p}} \log \frac{\left|\mathbf{H}_{\mathbf{0}}\right|}{\left|\mathbf{H}_{\mathbf{1}}\right|} & =\sqrt{p}\left[-\frac{1}{p} \log \left|\mathbf{H}_{\mathbf{0}}\right|-\left(\frac{1}{p} \log \left|\mathbf{H}_{\mathbf{1}}\right|\right)\right] \\
& =\sqrt{p}\left(\widetilde{V}_{0}+\log (1-\gamma)-\widetilde{V}_{1}-\log (1-\gamma)\right) \\
& =\sqrt{p}\left(\widetilde{V}_{0}-\widetilde{V}_{1}\right) \\
& =\frac{1}{\sqrt{p}} \log \left(\frac{n_{0}+p e_{00}}{n_{0}+p e_{10}}\right)^{n_{0}}\left(\frac{n_{1}+p e_{01}}{n_{1}+p e_{11}}\right)^{n_{1}}+\frac{1}{\sqrt{p}} \log \frac{\left|\mathbf{T}_{0}\right|}{\left|\mathbf{T}_{1}\right|} \\
& +\sqrt{p}(1-\gamma)\left(m_{10} e_{10}+m_{11} e_{11}-m_{00} e_{00}-m_{01} e_{01}\right)
\end{aligned}
$$


It is easy to derive the following transformations in order to use just one system of deterministic equivalence in our results.

$$
\begin{array}{r}
\mathbf{T}_{\mathbf{i}}=\frac{\mathbf{Q}_{\mathbf{i}}^{-1}}{1-\gamma} \\
e_{i 0}=\frac{\alpha_{i} \delta_{i} n_{0}}{p} \\
e_{i 1}=\frac{\beta_{i} \delta_{i} n_{0}}{p} \\
m_{i 0}=\frac{1}{(1-\gamma)\left(1+\alpha_{i} \delta_{i}\right)} \\
m_{i 1}=\frac{1}{(1-\gamma)\left(1+\frac{n_{0}}{n_{1}} \beta_{i} \delta_{i}\right)}
\end{array}
$$

Then, we can derive the following equivalence for $-\frac{1}{\sqrt{p}} \log \frac{\left|\mathbf{H}_{0}\right|}{\left|\mathbf{H}_{1}\right|}$

$$
\begin{aligned}
-\frac{1}{\sqrt{p}} \log \frac{\left|\mathbf{H}_{\mathbf{0}}\right|}{\left|\mathbf{H}_{\mathbf{1}}\right|} & =\frac{1}{\sqrt{p}} \log \left(\frac{1+\alpha_{0} \delta_{0}}{1+\alpha_{1} \delta_{1}}\right)^{n_{0}}\left(\frac{1+\beta_{0} \delta_{0}}{1+\beta_{1} \delta_{1}}\right)^{n_{1}}+\frac{1}{\sqrt{p}} \log \frac{\left|\mathbf{Q}_{1}\right|}{\left|\mathbf{Q}_{0}\right|} \\
& +\frac{1}{\sqrt{p}}\left[\frac{\alpha_{1} \delta_{1} n_{0}-\alpha_{0} \delta_{0} n_{0}}{\left(1+\alpha_{1} \delta_{1}\right)\left(1+\alpha_{0} \delta_{0}\right)}+\frac{\beta_{1} \delta_{1} n_{0}-\beta_{0} \delta_{0} n_{0}}{\left(1+\beta_{1} \delta_{1}\right)\left(1+\beta_{0} \delta_{0}\right)}\right]
\end{aligned}
$$

Next, we prove the equivalence for remaining terms of $\xi_{i}$, which is

$$
\frac{1}{\sqrt{p}}\left[\left(\boldsymbol{\mu}_{i}-\overline{\mathbf{x}}_{0}\right)^{T} \mathbf{H}_{0}\left(\boldsymbol{\mu}_{i}-\overline{\mathbf{x}}_{0}\right)-\left(\boldsymbol{\mu}_{i}-\overline{\mathbf{x}}_{1}\right)^{T} \mathbf{H}_{1}\left(\boldsymbol{\mu}_{i}-\overline{\mathbf{x}}_{1}\right)\right] \rightarrow \frac{1}{\sqrt{p}}\left[\delta_{0}-\bar{\delta}_{1}+(-1)^{i+1} \boldsymbol{\mu}^{T} \mathbf{Q}_{\mathbf{1}-\mathbf{i}} \boldsymbol{\mu}\right]
$$

To start with, we define $\mathbf{u}_{i}=\frac{\mathbf{1}_{n_{i} \times 1}}{n_{i}}$. By simple manipulation, we can show that $\overline{\mathbf{x}}_{i}=\boldsymbol{\mu}_{\boldsymbol{i}}+\mathbf{Y}_{i} \mathbf{u}_{i}$ Take $i=0$ for example.

$$
\begin{array}{r}
\left(\boldsymbol{\mu}_{0}-\overline{\mathbf{x}}_{0}\right)^{T} \mathbf{H}_{0}\left(\boldsymbol{\mu}_{0}-\overline{\mathbf{x}}_{0}\right)-\left(\boldsymbol{\mu}_{0}-\overline{\mathbf{x}}_{1}\right)^{T} \mathbf{H}_{1}\left(\boldsymbol{\mu}_{0}-\overline{\mathbf{x}}_{1}\right) \\
=\mathbf{u}_{0}^{T} \mathbf{Y}_{0}^{T} \mathbf{H}_{0} \mathbf{Y}_{0} \mathbf{u}_{0}-\boldsymbol{\mu}^{T} \mathbf{H}_{1} \boldsymbol{\mu}+2 \boldsymbol{\mu}^{T} \mathbf{H}_{1} \mathbf{Y}_{1} \mathbf{u}_{1}-\mathbf{u}_{1}^{T} \mathbf{Y}_{1}^{T} \mathbf{H}_{1} \mathbf{Y}_{1} \mathbf{u}_{1}
\end{array}
$$


Using (A.6), we have

$$
\boldsymbol{\mu}^{T} \mathbf{H}_{1} \boldsymbol{\mu}-\boldsymbol{\mu}^{T} \mathbf{Q}_{1} \boldsymbol{\mu} \stackrel{p}{\rightarrow} 0
$$

Before proving the remaining terms, we we first present some preliminary matrix manipulation. The eigen-decomposition of the matrix $n_{i} \mathbf{u}_{i} \mathbf{u}_{i}^{T}=\mathbf{U}_{i} \mathbf{D}_{i} \mathbf{U}_{i}^{T}$, where $\mathbf{D}_{i}=\operatorname{diag}\left(\left[1, \mathbf{0}_{\left(n_{i}-1\right) \times 1}\right]\right)$ and $\mathbf{U}_{i}$ is a unitary matrix. From the definition of $\widehat{\boldsymbol{\Sigma}}_{i}$, we can easily rewrite it as

$$
\begin{aligned}
\widehat{\boldsymbol{\Sigma}}_{i} & =\frac{1}{n_{i}-1}\left(\mathbf{Y}_{i} \mathbf{Y}_{i}^{T}-n_{i} \mathbf{Y}_{i} \mathbf{u}_{i} \mathbf{u}_{i}^{T} \mathbf{Y}_{i}^{T}\right) \\
& =\frac{1}{n_{i}-1} \mathbf{Y}_{i} \mathbf{U}_{i} \mathbf{U}_{i}^{T} \mathbf{Y}_{i}^{T}-\frac{1}{n_{i}-1} \underbrace{\mathbf{Y}_{i} \mathbf{U}_{i}}_{\widetilde{\mathbf{Y}}_{i}} \mathbf{D}_{i} \mathbf{U}_{i}^{T} \mathbf{Y}_{i}^{T} \\
& =\frac{1}{n_{i}-1} \widetilde{\mathbf{Y}}_{i} \widetilde{\mathbf{Y}}_{i}^{T}-\frac{1}{n_{i}-1} \widetilde{\mathbf{y}}_{i, 1} \widetilde{\mathbf{Y}}_{i, 1}^{T},
\end{aligned}
$$

where $\widetilde{\mathbf{y}}_{i, j}$ is the $j$ th column of $\widetilde{\mathbf{Y}}_{i}$. By the invariance of the Gaussian distribution by multiplication by a unitary matrix, $\widetilde{\mathbf{Y}}_{i}$ has the same distribution as $\mathbf{Y}_{i}$. Thus,

$\mathbf{H}_{i}=\left((1-\gamma) \mathbf{I}_{p}+\frac{\alpha_{i}}{n_{0}-2} \widetilde{\mathbf{Y}}_{0} \widetilde{\mathbf{Y}}_{0}^{T}+\frac{\beta_{i}}{n_{1}-2} \widetilde{\mathbf{Y}}_{1} \widetilde{\mathbf{Y}}_{1}^{T}-\frac{\alpha_{i}}{n_{0}-2} \widetilde{\mathbf{y}}_{01} \widetilde{\mathbf{y}}_{01}^{T}-\frac{\beta_{i}}{n_{1}-2} \widetilde{\mathbf{y}}_{11} \widetilde{\mathbf{y}}_{11}^{T}\right)^{-1}$

Therefore, we can see that $\widetilde{\mathbf{y}}_{i 1}$ is independent of $\mathbf{H}_{i}$. Moreover,

$$
\begin{aligned}
\mathbf{u}_{i}^{T} \mathbf{Y}_{i}^{T} \mathbf{H}_{i} \mathbf{Y}_{i} \mathbf{u}_{i} & =\operatorname{tr} \mathbf{Y}_{i} \mathbf{u}_{i} \mathbf{u}_{i}^{T} \mathbf{Y}_{i}^{T} \mathbf{H}_{i} \\
& =\frac{1}{n_{i}} \operatorname{tr} \widetilde{\mathbf{y}}_{i 1} \widetilde{\mathbf{y}}_{i}^{T} \mathbf{H}_{i} \\
& =\frac{1}{n_{i}} \widetilde{\mathbf{y}}_{i 1}^{T} \mathbf{H}_{i} \widetilde{\mathbf{y}}_{i 1}
\end{aligned}
$$


By independence of $\widetilde{\mathbf{y}}_{i 1}$ and $\mathbf{H}_{i}$ and by application of the trace lemma 14, Theorem

$$
\mathbf{u}_{i} \mathbf{Y}_{i}^{T} \mathbf{H}_{i} \mathbf{Y}_{i} \mathbf{u}_{i}-\frac{1}{n_{i}} \operatorname{tr} \boldsymbol{\Sigma}_{i} \mathbf{H}_{i} \stackrel{\text { a.s. }}{\longrightarrow} 0
$$

Again, using (A.6), we derive the final equivalence

$$
\frac{1}{n_{i}} \operatorname{tr} \boldsymbol{\Sigma}_{i} \mathbf{H}_{i}-\frac{1}{n_{i}} \operatorname{tr} \boldsymbol{\Sigma}_{i} \mathbf{Q}_{i} \rightarrow 0
$$

Similarly, by independence of $\widetilde{y}_{i 1}$ and $\mathbf{H}_{1}$, it is easy to derive that

$$
\boldsymbol{\mu}^{T} \mathbf{H}_{1} \mathbf{Y}_{1} \mathbf{u}_{1} \stackrel{\text { a.s. }}{\longrightarrow} 0 \text {. }
$$

The proof for $i=1$ follows exactly the same procedure. Finally, we can conclude that

$$
\frac{1}{\sqrt{p}}\left[\left(\boldsymbol{\mu}_{i}-\overline{\mathbf{x}}_{0}\right)^{T} \mathbf{H}_{0}\left(\boldsymbol{\mu}_{i}-\overline{\mathbf{x}}_{0}\right)-\left(\boldsymbol{\mu}_{i}-\overline{\mathbf{x}}_{1}\right)^{T} \mathbf{H}_{1}\left(\boldsymbol{\mu}_{i}-\overline{\mathbf{x}}_{1}\right)\right] \rightarrow \frac{1}{\sqrt{p}}\left[\delta_{0}-\bar{\delta}_{1}+(-1)^{i+1} \boldsymbol{\mu}^{T} \mathbf{Q}_{\mathbf{1 - i}} \boldsymbol{\mu}\right]
$$

\section{A.0.2 Proof of $\mathrm{A} .8$}

The proof for convergence of $\frac{1}{\sqrt{p}} \operatorname{tr} \mathbf{B}_{i}$ is straightforward.

$$
\begin{aligned}
\frac{1}{\sqrt{p}} \operatorname{tr} \mathbf{B}_{i} & =\frac{1}{\sqrt{p}} \operatorname{tr} \boldsymbol{\Sigma}_{i}^{1 / 2}\left(\mathbf{H}_{1}-\mathbf{H}_{0}\right) \boldsymbol{\Sigma}_{i}^{1 / 2} \\
& =\frac{1}{\sqrt{p}} \operatorname{tr} \boldsymbol{\Sigma}_{i}\left(\mathbf{H}_{1}-\mathbf{H}_{0}\right) \\
& =\frac{1}{\sqrt{p}} \operatorname{tr} \boldsymbol{\Sigma}_{i} \mathbf{H}_{1}-\frac{1}{\sqrt{p}} \operatorname{tr} \boldsymbol{\Sigma}_{i} \mathbf{H}_{0}
\end{aligned}
$$


Since $\mathbf{Q}_{i}-\mathbf{H}_{i} \rightarrow 0$, we have

$$
\frac{1}{p} \operatorname{tr} \boldsymbol{\Sigma}_{i} \mathbf{H}_{i}-\frac{1}{p} \operatorname{tr} \boldsymbol{\Sigma}_{i} \mathbf{Q}_{i}=\mathcal{O}\left(\frac{1}{p}\right)
$$

Therefore, we get convergence in probability of $\frac{1}{\sqrt{p}} \operatorname{tr} \mathbf{B}_{i}$ to $\bar{b}_{i}$,

$$
\frac{1}{\sqrt{p}} \operatorname{tr} \mathbf{B}_{i}-\frac{1}{\sqrt{p}} \operatorname{tr} \boldsymbol{\Sigma}_{i}\left(\mathbf{Q}_{1}-\mathbf{Q}_{0}\right)=\mathcal{O}\left(\frac{1}{\sqrt{p}}\right)
$$

\section{A.0.3 Proof of A.9}

The proof for convergence of $\frac{1}{p} \operatorname{tr} \mathbf{B}_{i}^{2}$ is a little bit complex. The main principle is to use trace lemma and inversion lemma.

$$
\begin{aligned}
\frac{1}{p} \operatorname{tr} \mathbf{B}_{i}^{2} & =\frac{1}{p} \operatorname{tr} \boldsymbol{\Sigma}_{i}\left(\mathbf{H}_{1}-\mathbf{H}_{0}\right) \boldsymbol{\Sigma}_{i}\left(\mathbf{H}_{1}-\mathbf{H}_{0}\right) \\
& =\frac{1}{p} \operatorname{tr} \boldsymbol{\Sigma}_{i} \mathbf{H}_{1} \boldsymbol{\Sigma}_{i} \mathbf{H}_{1}+\frac{1}{p} \operatorname{tr} \boldsymbol{\Sigma}_{i} \mathbf{H}_{0} \boldsymbol{\Sigma}_{i} \mathbf{H}_{0}-\frac{2}{p} \operatorname{tr} \boldsymbol{\Sigma}_{i} \mathbf{H}_{0} \boldsymbol{\Sigma}_{i} \mathbf{H}_{1} .
\end{aligned}
$$

We first prove the convergence for $\frac{1}{p} \operatorname{tr} \mathbf{A} \mathbf{H}_{0} \mathbf{B} \mathbf{H}_{1}$ where $\mathbf{A}$ and $\mathbf{B}$ are arbitrary matrix with finite spectral norms. To start with, we first rewrite $\mathbf{H}_{i}$ as follows,

$$
\begin{aligned}
& \widehat{\boldsymbol{\Sigma}}_{i}=\frac{\mathbf{Y}_{i} \mathbf{Y}_{i}^{T}}{n_{i}} \\
& =\frac{1}{n_{i}} \sum_{k=1}^{n_{i}} \mathbf{y}_{i k} \mathbf{y}_{i k}^{T} \\
& =\frac{1}{n_{i}} \mathbf{y}_{i j} \mathbf{y}_{i j}^{T}+\frac{1}{n_{i}} \sum_{k \neq j} \mathbf{y}_{i k} \mathbf{y}_{i k}^{T} \\
& \mathbf{H}_{i}=[\frac{\alpha_{i}}{n_{0}} \mathbf{y}_{0 j} \mathbf{y}_{0 j}^{T}+\underbrace{\frac{\alpha_{i}}{n_{0}} \sum_{k \neq j} \mathbf{y}_{0 k} \mathbf{y}_{0 k}^{T}+\beta_{i} \widehat{\mathbf{\Sigma}}_{1}+(1-\gamma) \mathbf{I}_{p}}_{\mathbf{A}_{i}}]^{-1}
\end{aligned}
$$


Then, by using Sherman-Morrison inversion Lemma [15], we have

$$
\mathbf{H}_{i}=\mathbf{A}_{i}^{-1}-\frac{\frac{\alpha_{i}}{n_{0}} \mathbf{A}_{i}^{-1} \mathbf{y}_{0 j} \mathbf{y}_{0 j}^{T} \mathbf{A}_{i}^{-1}}{1+\frac{\alpha_{i}}{n_{0}} \mathbf{y}_{0 j}^{T} \mathbf{A}_{i}^{-1} \mathbf{y}_{0 j}}
$$

For $\frac{1}{n_{0}} \mathbf{y}_{0 j}^{T} \mathbf{A}_{i}^{-1} \mathbf{y}_{0 j}$, by using trace lemmma again, we can get

$$
\frac{1}{n_{0}} \mathbf{y}_{0 j}^{T} \mathbf{A}_{i}^{-1} \mathbf{y}_{0 j} \rightarrow \frac{1}{n_{0}} \operatorname{tr} \boldsymbol{\Sigma}_{0} \mathbf{A}_{i}^{-1} \rightarrow \frac{1}{n_{0}} \operatorname{tr} \boldsymbol{\Sigma}_{0} \mathbf{Q}_{i}=\delta_{i} .
$$

Therefore,

$$
\mathbf{H}_{i} \rightarrow \mathbf{A}_{i}^{-1}-\frac{\frac{\alpha_{i}}{n_{0}} \mathbf{A}_{i}^{-1} \mathbf{y}_{0 j} \mathbf{y}_{0 j}^{T} \mathbf{A}_{i}^{-1}}{1+\alpha_{i} \delta_{i}}
$$

To start with, we write

$$
\frac{1}{p} \operatorname{tr} \mathbf{A H}_{0} \mathbf{B} \mathbf{H}_{1}=\frac{1}{p} \operatorname{tr} \mathbf{A H}_{0} \mathbf{B} \mathbf{Q}_{1}+\frac{1}{p} \operatorname{tr} \mathbf{A} \mathbf{H}_{0} \mathbf{B}\left(\mathbf{H}_{1}-\mathbf{Q}_{1}\right)
$$

Then, by using resolvent identity and write $\mathbf{Q}_{1}=\left[\widetilde{\delta}_{0} \boldsymbol{\Sigma}_{0}+\widetilde{\delta}_{1} \boldsymbol{\Sigma}_{1}+(1-\gamma) \mathbf{I}_{p}\right]^{-1}$, we have

$$
\begin{aligned}
\frac{1}{p} \operatorname{tr} \mathbf{A H}_{0} \mathbf{B}\left(\mathbf{H}_{1}-\mathbf{Q}_{1}\right) & =\frac{1}{p} \operatorname{tr} \mathbf{A H}_{0} \mathbf{B} \mathbf{H}_{1}\left(\mathbf{Q}_{1}^{-1}-\mathbf{H}_{1}^{-1}\right) \mathbf{Q}_{1} \\
& =\frac{1}{p} \operatorname{tr} \mathbf{A} \mathbf{H}_{0} \mathbf{B} \mathbf{H}_{1}\left(\mathbf{Q}_{1}^{-1}-\alpha_{1} \widehat{\boldsymbol{\Sigma}}_{0}-\beta 1 \widehat{\boldsymbol{\Sigma}}_{1}-(1-\gamma) \mathbf{I}_{p}\right) \mathbf{Q}_{1} \\
& =\frac{1}{p} \operatorname{tr} \mathbf{A} \mathbf{H}_{0} \mathbf{B} \mathbf{H}_{1}\left(\widetilde{\delta}_{0} \boldsymbol{\Sigma}_{0}+\widetilde{\delta}_{1} \boldsymbol{\Sigma}_{1}-\alpha_{1} \widehat{\boldsymbol{\Sigma}}_{0}-\beta 1 \widehat{\Sigma}_{1}\right) \mathbf{Q}_{1}
\end{aligned}
$$




$$
\begin{aligned}
\frac{1}{p} \operatorname{tr} \mathbf{A} \mathbf{H}_{0} \mathbf{B} \mathbf{H}_{1} \alpha_{1} \widehat{\Sigma}_{0} \mathbf{Q}_{1} & =\frac{\alpha_{1}}{p} \sum_{k=1}^{n_{0}} \frac{1}{n_{0}} \mathbf{A} \mathbf{H}_{0} \mathbf{B} \mathbf{H}_{1} \mathbf{y}_{0 k} \mathbf{y}_{0 k}^{T} \mathbf{Q}_{1} \\
& =\frac{\alpha_{1}}{p} \sum_{k=1}^{n_{0}} \frac{1}{n_{0}} \mathbf{y}_{0 k}^{T} \mathbf{Q}_{1} \mathbf{A} \mathbf{H}_{0} \mathbf{B} \mathbf{H}_{1} \mathbf{y}_{0 k} \\
& =\frac{\alpha_{1}}{p} \mathbf{y}_{0 k}^{T} \mathbf{Q}_{1} \mathbf{A}\left(\mathbf{A}_{0}^{-1}-\frac{\frac{\alpha_{0}}{n_{0}} \mathbf{A}_{0}^{-1} \mathbf{y}_{0 k} \mathbf{y}_{0 k}^{T} \mathbf{A}_{0}^{-1}}{1+\alpha_{0} \delta_{0}}\right) \mathbf{B} \\
& \left(\mathbf{A}_{1}^{-1}-\frac{\left.\frac{\alpha_{1}}{n_{0}} \mathbf{A}_{1}^{-1} \mathbf{y}_{0 k} \mathbf{y}_{0 k}^{T} \mathbf{A}_{1}^{-1}\right)}{1+\alpha_{1} \delta_{1}}\right) \mathbf{y}_{0 k} \\
& =\frac{\alpha_{1}}{p} \mathbf{y}_{0 k}^{T} \mathbf{Q}_{1} \mathbf{A} \mathbf{A}_{0}^{-1} \mathbf{B} \mathbf{A}_{1}^{-1} \mathbf{y}_{0 k}-\frac{\alpha_{1}}{p} \mathbf{y}_{0 k}^{T} \mathbf{Q}_{1} \mathbf{A} \mathbf{A}_{0}^{-1} \mathbf{B} \mathbf{A}_{1}^{-1} \mathbf{y}_{0 k} \frac{\alpha_{1}}{n_{0}} \frac{\mathbf{y}_{0 k}^{T} \mathbf{A}_{1}^{-1} \mathbf{y}_{0 k}}{1+\alpha_{1} \delta_{1}} \\
& -\frac{\alpha_{1}}{p} \mathbf{y}_{0 k}^{T} \mathbf{Q}_{1} \mathbf{A} \mathbf{A}_{0}^{-1} \mathbf{y}_{0 k} \frac{\alpha_{0}}{n_{0}} \frac{\mathbf{y}_{0 k}^{T} \mathbf{A}_{0}^{-1} \mathbf{B} \mathbf{A}_{1}^{-1} \mathbf{y}_{0 k}}{1+\alpha_{0} \delta_{0}} \\
& +\frac{\alpha_{1}}{p} \frac{\alpha_{0}}{n_{0}}\left(\mathbf{y}_{0 k}^{T} \mathbf{Q}_{1} \mathbf{A} \mathbf{A}_{0}^{-1} \mathbf{y}_{0 k}\right) \frac{\alpha_{1}}{n_{0}} \frac{\mathbf{y}_{0 k}^{T} \mathbf{A}_{0}^{-1} \mathbf{B} \mathbf{A}_{1}^{-1} \mathbf{y}_{0 k}}{1+\alpha_{0} \delta_{0}} \frac{\mathbf{y}_{0 k}^{T} \mathbf{A}_{1}^{-1} \mathbf{y}_{0 k}}{1+\alpha_{1} \delta_{1}}
\end{aligned}
$$

Then, by application of trace lemma, we get the final simplified equivalence for the above expanding terms as follows:

$$
\begin{aligned}
\frac{1}{p} \operatorname{tr} \mathbf{A} \mathbf{H}_{0} \mathbf{B} \mathbf{H}_{1} \alpha_{1} \widehat{\mathbf{\Sigma}}_{0} \mathbf{Q}_{1} & =\frac{1}{p} \frac{\alpha_{1}}{1+\alpha_{1} \delta_{1}} \operatorname{tr} \boldsymbol{\Sigma}_{0} \mathbf{Q}_{1} \mathbf{A} \mathbf{H}_{0} \mathbf{B} \mathbf{H}_{1} \\
& -\frac{\alpha_{0} \alpha_{1}}{p n_{0}\left(1+\alpha_{0} \delta_{0}\right)\left(1+\alpha_{1} \delta_{1}\right)} \operatorname{tr} \boldsymbol{\Sigma}_{0} \mathbf{Q}_{1} \mathbf{A} \mathbf{H}_{0} \operatorname{tr} \boldsymbol{\Sigma}_{0} \mathbf{H}_{0} \mathbf{B} \mathbf{H}_{1}
\end{aligned}
$$

Moreover, following the same steps as proving convergence of $\frac{1}{p} \operatorname{tr} \mathbf{A H}_{0} \mathbf{B} \mathbf{H}_{1} \beta_{1} \widehat{\Sigma}_{1} \mathbf{Q}_{1}$, it is easy to show that

$$
\begin{aligned}
\frac{1}{p} \operatorname{tr} \mathbf{A} \mathbf{H}_{0} \mathbf{B} \mathbf{H}_{1} \beta_{1} \widehat{\boldsymbol{\Sigma}}_{1} \mathbf{Q}_{1} & =\frac{1}{p} \frac{\beta_{1}}{1+\beta_{1} \bar{\delta}_{1}} \operatorname{tr} \boldsymbol{\Sigma}_{1} \mathbf{Q}_{1} \mathbf{A} \mathbf{H}_{0} \mathbf{B} \mathbf{H}_{1} \\
& -\frac{\beta_{0} \beta_{1}}{p n_{1}\left(1+\beta_{0} \bar{\delta}_{0}\right)\left(1+\beta_{1} \bar{\delta}_{1}\right)} \operatorname{tr} \boldsymbol{\Sigma}_{1} \mathbf{Q}_{1} \mathbf{A} \mathbf{H}_{0} \operatorname{tr} \boldsymbol{\Sigma}_{1} \mathbf{H}_{0} \mathbf{B} \mathbf{H}_{1}
\end{aligned}
$$


We know $\widetilde{\delta}_{0}=\frac{\alpha_{1}}{1+\alpha_{1} \delta_{1}}, \widetilde{\delta}_{1}=\frac{\beta_{1}}{1+\beta_{1} \bar{\delta}_{1}}, \tilde{\delta}_{00}=\frac{\alpha_{0}}{1+\alpha_{0} \delta_{0}}$ and $\tilde{\delta}_{11}=\frac{\beta_{0}}{1+\beta_{0} \bar{\delta}_{0}}(\bar{\delta}=\delta)$ therefore,

$$
\begin{aligned}
\frac{1}{p} \operatorname{tr} \mathbf{A H}_{0} \mathbf{B} \mathbf{H}_{1} & =\frac{1}{p} \operatorname{tr} \mathbf{A} \mathbf{H}_{0} \mathbf{B} \mathbf{Q}_{1}+\frac{\alpha_{0} \widetilde{\delta}_{0}}{p n_{0}\left(1+\alpha_{0} \delta_{0}\right)} \operatorname{tr} \mathbf{\Sigma}_{0} \mathbf{Q}_{1} \mathbf{A} \mathbf{H}_{0} \operatorname{tr} \mathbf{\Sigma}_{0} \mathbf{H}_{0} \mathbf{B} \mathbf{H}_{1} \\
& +\frac{\beta_{0} \widetilde{\delta}_{1}}{p n_{1}\left(1+\beta_{0} \bar{\delta}_{0}\right)} \operatorname{tr} \boldsymbol{\Sigma}_{1} \mathbf{Q}_{1} \mathbf{A} \mathbf{H}_{0} \operatorname{tr} \boldsymbol{\Sigma}_{1} \mathbf{H}_{0} \mathbf{B} \mathbf{H}_{1}
\end{aligned}
$$

To derive the equivalence for $\frac{1}{p} \operatorname{tr} \boldsymbol{\Sigma}_{0} \mathbf{H}_{0} \boldsymbol{\Sigma}_{0} \mathbf{H}_{1}$, we first take $\mathbf{A}=\boldsymbol{\Sigma}_{0}$, $\mathbf{B}=\boldsymbol{\Sigma}_{0}$ and then take $\mathbf{A}=\boldsymbol{\Sigma}_{1}, \mathbf{B}=\boldsymbol{\Sigma}_{0}$.

$$
\begin{aligned}
\frac{1}{p} \operatorname{tr} \boldsymbol{\Sigma}_{0} \mathbf{H}_{0} \boldsymbol{\Sigma}_{0} \mathbf{H}_{1}= & \frac{1}{p} \operatorname{tr} \boldsymbol{\Sigma}_{0} \mathbf{H}_{0} \boldsymbol{\Sigma}_{0} \mathbf{Q}_{1}+\frac{\alpha_{0} \widetilde{\delta}_{0}}{p n_{0}\left(1+\alpha_{0} \delta_{0}\right)} \operatorname{tr} \boldsymbol{\Sigma}_{0} \mathbf{Q}_{1} \boldsymbol{\Sigma}_{0} \mathbf{H}_{0} \operatorname{tr} \boldsymbol{\Sigma}_{0} \mathbf{H}_{0} \boldsymbol{\Sigma}_{0} \mathbf{H}_{1} \\
& +\frac{\beta_{0} \widetilde{\delta}_{1}}{p n_{1}\left(1+\beta_{0} \bar{\delta}_{0}\right)} \operatorname{tr} \boldsymbol{\Sigma}_{1} \mathbf{Q}_{1} \boldsymbol{\Sigma}_{0} \mathbf{H}_{0} \operatorname{tr} \boldsymbol{\Sigma}_{1} \mathbf{H}_{0} \boldsymbol{\Sigma}_{0} \mathbf{H}_{1}
\end{aligned}
$$

$$
\begin{aligned}
\frac{1}{p} \operatorname{tr} \boldsymbol{\Sigma}_{1} \mathbf{H}_{0} \boldsymbol{\Sigma}_{0} \mathbf{H}_{1}= & \frac{1}{p} \operatorname{tr} \boldsymbol{\Sigma}_{1} \mathbf{H}_{0} \boldsymbol{\Sigma}_{0} \mathbf{Q}_{1}+\frac{\alpha_{0} \widetilde{\delta}_{0}}{p n_{0}\left(1+\alpha_{0} \delta_{0}\right)} \operatorname{tr} \boldsymbol{\Sigma}_{0} \mathbf{Q}_{1} \boldsymbol{\Sigma}_{1} \mathbf{H}_{0} \operatorname{tr} \boldsymbol{\Sigma}_{0} \mathbf{H}_{0} \boldsymbol{\Sigma}_{0} \mathbf{H}_{1} \\
& +\frac{\beta_{0} \widetilde{\delta}_{1}}{p n_{1}\left(1+\beta_{0} \bar{\delta}_{0}\right)} \operatorname{tr} \boldsymbol{\Sigma}_{1} \mathbf{Q}_{1} \boldsymbol{\Sigma}_{0} \mathbf{H}_{0} \operatorname{tr} \boldsymbol{\Sigma}_{1} \mathbf{H}_{0} \boldsymbol{\Sigma}_{1} \mathbf{H}_{1}
\end{aligned}
$$

By combining equation A.16 and A.17, we can solve $\frac{1}{p} \operatorname{tr} \boldsymbol{\Sigma}_{0} \mathbf{H}_{0} \boldsymbol{\Sigma}_{0} \mathbf{H}_{1}$.

For solving $\frac{1}{p} \operatorname{tr} \boldsymbol{\Sigma}_{1} \mathbf{H}_{0} \boldsymbol{\Sigma}_{1} \mathbf{H}_{1}$, we first take $\mathbf{A}=\boldsymbol{\Sigma}_{1}, \mathbf{B}=\boldsymbol{\Sigma}_{1}$ and then take $\mathbf{A}=\boldsymbol{\Sigma}_{0}$,

$$
\begin{aligned}
\mathbf{B}=\boldsymbol{\Sigma}_{1} . & \\
\frac{1}{p} \operatorname{tr} \boldsymbol{\Sigma}_{1} \mathbf{H}_{0} \boldsymbol{\Sigma}_{1} \mathbf{H}_{1}= & \frac{1}{p} \operatorname{tr} \boldsymbol{\Sigma}_{1} \mathbf{H}_{0} \boldsymbol{\Sigma}_{1} \mathbf{Q}_{1}+\frac{\alpha_{0} \widetilde{\delta}_{0}}{p n_{0}\left(1+\alpha_{0} \delta_{0}\right)} \operatorname{tr} \boldsymbol{\Sigma}_{0} \mathbf{Q}_{1} \boldsymbol{\Sigma}_{1} \mathbf{H}_{0} \operatorname{tr} \boldsymbol{\Sigma}_{0} \mathbf{H}_{0} \boldsymbol{\Sigma}_{1} \mathbf{H}_{1} \\
& +\frac{\beta_{0} \widetilde{\delta}_{1}}{p n_{1}\left(1+\beta_{0} \bar{\delta}_{0}\right)} \operatorname{tr} \boldsymbol{\Sigma}_{1} \mathbf{Q}_{1} \boldsymbol{\Sigma}_{1} \mathbf{H}_{0} \operatorname{tr} \boldsymbol{\Sigma}_{1} \mathbf{H}_{0} \boldsymbol{\Sigma}_{1} \mathbf{H}_{1} \\
\frac{1}{p} \operatorname{tr} \boldsymbol{\Sigma}_{0} \mathbf{H}_{0} \boldsymbol{\Sigma}_{1} \mathbf{H}_{1}= & \frac{1}{p} \operatorname{tr} \boldsymbol{\Sigma}_{0} \mathbf{H}_{0} \boldsymbol{\Sigma}_{1} \mathbf{Q}_{1}+\frac{\alpha_{0} \widetilde{\delta}_{0}}{p n_{0}\left(1+\alpha_{0} \delta_{0}\right)} \operatorname{tr} \boldsymbol{\Sigma}_{0} \mathbf{Q}_{1} \boldsymbol{\Sigma}_{0} \mathbf{H}_{0} \operatorname{tr} \boldsymbol{\Sigma}_{0} \mathbf{H}_{0} \boldsymbol{\Sigma}_{1} \mathbf{H}_{1} \\
& +\frac{\beta_{0} \widetilde{\delta}_{1}}{p n_{1}\left(1+\beta_{0} \bar{\delta}_{0}\right)} \operatorname{tr} \boldsymbol{\Sigma}_{1} \mathbf{Q}_{1} \boldsymbol{\Sigma}_{0} \mathbf{H}_{0} \operatorname{tr} \boldsymbol{\Sigma}_{1} \mathbf{H}_{0} \boldsymbol{\Sigma}_{1} \mathbf{H}_{1}
\end{aligned}
$$


Similarly, by combing equation A.18 and A.19, we can solve $\frac{1}{p} \operatorname{tr} \boldsymbol{\Sigma}_{1} \mathbf{H}_{0} \boldsymbol{\Sigma}_{1} \mathbf{H}_{1}$.

Finally, we generalize the following equivalence for $\frac{1}{p} \operatorname{tr} \boldsymbol{\Sigma}_{i} \mathbf{H}_{0} \boldsymbol{\Sigma}_{i} \mathbf{H}_{1}$ as follows.

$\frac{1}{p} \frac{\operatorname{tr} \Sigma_{i} \mathbf{Q}_{0} \Sigma_{i} \mathbf{Q}_{1}\left(1-\frac{\widetilde{\delta}_{j} \widetilde{\delta}_{j j}}{n_{j}} \operatorname{tr} \boldsymbol{\Sigma}_{j} \mathbf{Q}_{1} \boldsymbol{\Sigma}_{j} \mathbf{Q}_{0}\right)+\frac{\widetilde{\delta}_{j} \widetilde{\delta}_{j j}}{n_{j}} \operatorname{tr} \boldsymbol{\Sigma}_{1} \mathbf{Q}_{1} \boldsymbol{\Sigma}_{0} \mathbf{Q}_{0} \operatorname{tr} \boldsymbol{\Sigma}_{0} \mathbf{Q}_{1} \boldsymbol{\Sigma}_{1} \mathbf{Q}_{0}}{\left(1-\frac{\widetilde{\delta}_{1} \widetilde{\delta}_{11}}{n_{1}} \operatorname{tr} \boldsymbol{\Sigma}_{1} \mathbf{Q}_{1} \boldsymbol{\Sigma}_{1} \mathbf{Q}_{0}\right)\left(1-\frac{\widetilde{\delta}_{0} \widetilde{\delta}_{00}}{n_{0}} \operatorname{tr} \boldsymbol{\Sigma}_{0} \mathbf{Q}_{1} \boldsymbol{\Sigma}_{0} \mathbf{Q}_{0}\right)-\frac{\widetilde{\delta}_{0} \widetilde{\delta}_{00} \widetilde{\delta}_{1} \widetilde{\delta}_{11}}{n_{0} n_{1}} \operatorname{tr} \boldsymbol{\Sigma}_{1} \mathbf{Q}_{1} \boldsymbol{\Sigma}_{0} \mathbf{Q}_{0} \operatorname{tr} \boldsymbol{\Sigma}_{0} \mathbf{Q}_{1} \boldsymbol{\Sigma}_{1} \mathbf{Q}_{0}}$

Using the same technique, we can easily derive the convergence for $\frac{1}{p} \operatorname{tr} \boldsymbol{\Sigma}_{i} \mathbf{H}_{0} \boldsymbol{\Sigma}_{i} \mathbf{H}_{0}$ and $\frac{1}{p} \operatorname{tr} \boldsymbol{\Sigma}_{i} \mathbf{H}_{1} \boldsymbol{\Sigma}_{i} \mathbf{H}_{1}$.

$$
\begin{aligned}
& \frac{1}{p} \operatorname{tr} \boldsymbol{\Sigma}_{i} \mathbf{H}_{0} \boldsymbol{\Sigma}_{i} \mathbf{H}_{0} \rightarrow \frac{1}{p} \frac{\operatorname{tr} \boldsymbol{\Sigma}_{i} \mathbf{Q}_{0} \boldsymbol{\Sigma}_{i} \mathbf{Q}_{0}\left(1-\frac{\widetilde{\delta}_{j j}^{2}}{n_{j}} \operatorname{tr} \boldsymbol{\Sigma}_{j} \mathbf{Q}_{0} \boldsymbol{\Sigma}_{j} \mathbf{Q}_{0}\right)+\frac{\widetilde{\delta}_{j j}^{2}}{n_{j}}\left(\operatorname{tr} \boldsymbol{\Sigma}_{1} \mathbf{Q}_{0} \boldsymbol{\Sigma}_{0} \mathbf{Q}_{0}\right)^{2}}{\left(1-\frac{\widetilde{\delta}_{11}^{2}}{n_{1}} \operatorname{tr} \boldsymbol{\Sigma}_{1} \mathbf{Q}_{0} \boldsymbol{\Sigma}_{1} \mathbf{Q}_{0}\right)\left(1-\frac{\widetilde{\delta}_{00}^{2}}{n_{0}} \operatorname{tr} \boldsymbol{\Sigma}_{0} \mathbf{Q}_{0} \boldsymbol{\Sigma}_{0} \mathbf{Q}_{0}\right)-\frac{\widetilde{\delta}_{00}^{2} \widetilde{\delta}_{11}^{2}\left(\operatorname{tr} \boldsymbol{\Sigma}_{1} \mathbf{Q}_{0} \boldsymbol{\Sigma}_{0} \mathbf{Q}_{0}\right)^{2}}{n_{0} n_{1}}} \\
& \frac{1}{p} \operatorname{tr} \boldsymbol{\Sigma}_{i} \mathbf{H}_{1} \boldsymbol{\Sigma}_{i} \mathbf{H}_{1} \rightarrow \frac{1}{p} \frac{\operatorname{tr} \boldsymbol{\Sigma}_{i} \mathbf{Q}_{1} \boldsymbol{\Sigma}_{i} \mathbf{Q}_{1}\left(1-\frac{\widetilde{\delta}_{j}^{2}}{n_{j}} \operatorname{tr} \boldsymbol{\Sigma}_{j} \mathbf{Q}_{1} \boldsymbol{\Sigma}_{j} \mathbf{Q}_{1}\right)+\frac{\widetilde{\delta}_{j}^{2}}{n_{j}}\left(\operatorname{tr} \boldsymbol{\Sigma}_{1} \mathbf{Q}_{1} \boldsymbol{\Sigma}_{0} \mathbf{Q}_{1}\right)^{2}}{\left(1-\frac{\widetilde{\delta}_{1}^{2}}{n_{1}} \operatorname{tr} \boldsymbol{\Sigma}_{1} \mathbf{Q}_{1} \boldsymbol{\Sigma}_{1} \mathbf{Q}_{1}\right)\left(1-\frac{\widetilde{\delta}_{0}^{2}}{n_{0}} \operatorname{tr} \boldsymbol{\Sigma}_{0} \mathbf{Q}_{1} \boldsymbol{\Sigma}_{0} \mathbf{Q}_{1}\right)-\frac{\widetilde{\delta}_{0}^{2} \widetilde{\delta}_{1}^{2}}{n_{0} n_{1}}\left(\operatorname{tr} \boldsymbol{\Sigma}_{1} \mathbf{Q}_{1} \boldsymbol{\Sigma}_{0} \mathbf{Q}_{1}\right)^{2}}
\end{aligned}
$$

Under assumption 4 , it is easy to derive the following equivalence between $\operatorname{tr} \boldsymbol{\Sigma}_{j} \mathbf{Q}_{p} \boldsymbol{\Sigma}_{k} \mathbf{Q}_{q}$ and $\operatorname{tr} \boldsymbol{\Sigma}_{m} \mathbf{Q}_{s} \boldsymbol{\Sigma}_{n} \mathbf{Q}_{t}$ for $j, k \in\{0,1\}, m, n \in\{0,1\}, p, q \in\{0,1\}$ and $s, t \in\{0,1\}$

$$
\operatorname{tr} \boldsymbol{\Sigma}_{j} \mathbf{Q}_{p} \boldsymbol{\Sigma}_{k} \mathbf{Q}_{q}=\operatorname{tr} \boldsymbol{\Sigma}_{m} \mathbf{Q}_{s} \boldsymbol{\Sigma}_{n} \mathbf{Q}_{t}
$$

Therefore, by using the above equivalence, we can further simplified $\frac{1}{p} \operatorname{tr} \mathbf{B}_{i}^{2}$.

\section{A.0.4 Proof of A.10}

$$
\mathbf{y}_{i}=\boldsymbol{\Sigma}_{i}^{1 / 2}\left[\mathbf{H}_{1}\left(\boldsymbol{\mu}_{i}-\overline{\mathbf{x}}_{1}\right)-\mathbf{H}_{0}\left(\boldsymbol{\mu}_{i}-\overline{\mathbf{x}}_{0}\right)\right]
$$

The proof for convergence of $\frac{1}{p} \mathbf{y}_{i}^{T} \mathbf{y}_{i}$ is similar to proof for B.4. By using the independence of $\widetilde{\mathbf{y}}_{i 1}$ and $\mathbf{H}_{i}$ and by simple application of trace lemma, It is easy to show 
that

$$
\frac{1}{p} \mathbf{y}_{i}^{T} \mathbf{y}_{i}-\frac{1}{p} \boldsymbol{\mu}^{T} \mathbf{H}_{1-i} \boldsymbol{\Sigma}_{i} \mathbf{H}_{1-i} \boldsymbol{\mu}-\frac{1}{p n_{1}} \operatorname{tr} \boldsymbol{\Sigma}_{1} \mathbf{H}_{1} \boldsymbol{\Sigma}_{i} \mathbf{H}_{1}-\frac{1}{p n_{0}} \operatorname{tr} \boldsymbol{\Sigma}_{0} \mathbf{H}_{0} \boldsymbol{\Sigma}_{i} \mathbf{H}_{0} \stackrel{p}{\rightarrow} 0
$$




\section{B Proof of Theorem 3}

The proof consists in proving the following convergences:

$$
\begin{aligned}
& \widehat{\xi}_{i}-\xi_{i} \stackrel{p}{\rightarrow} 0 . \\
& \widehat{b}_{i}-b_{i} \stackrel{p}{\rightarrow} 0 . \\
& \widehat{B}_{i}-B_{i} \stackrel{p}{\rightarrow} 0 .
\end{aligned}
$$

\section{B.0.1 Proof of B.1}

Applying the same technique as in proof of 3.2 , we have

$\frac{1}{\sqrt{p}}\left[\left(\boldsymbol{\mu}_{i}-\overline{\mathbf{x}}_{0}\right)^{T} \mathbf{H}_{0}\left(\boldsymbol{\mu}_{i}-\overline{\mathbf{x}}_{0}\right)-\left(\boldsymbol{\mu}_{i}-\overline{\mathbf{x}}_{1}\right)^{T} \mathbf{H}_{1}\left(\boldsymbol{\mu}_{i}-\overline{\mathbf{x}}_{1}\right)\right]-\frac{1}{\sqrt{p}}(-1)^{i+1} \boldsymbol{\mu}^{T} \mathbf{H}_{1-\mathbf{i}} \boldsymbol{\mu} \stackrel{p}{\rightarrow} 0$

It is easy to prove that

$$
\frac{1}{\sqrt{p}}(-1)^{i+1} \boldsymbol{\mu}^{T} \mathbf{H}_{\mathbf{1}-\mathbf{i}} \boldsymbol{\mu}-\frac{1}{\sqrt{p}}(-1)^{i+1}\left(\overline{\mathbf{x}}_{0}-\overline{\mathbf{x}}_{1}\right)^{T} \mathbf{H}_{\mathbf{1 - i}}\left(\overline{\mathbf{x}}_{0}-\overline{\mathbf{x}}_{1}\right) . \stackrel{p}{\rightarrow} 0
$$




\section{B.0.2 Proof of $B .2$}

The proof for $(\mathrm{B} .2)$ is again a combined application of trace lemma and inversion lemma as we did in 3.4. Take $i=0$ as example.

$$
\begin{aligned}
& \frac{1}{\sqrt{p}} \operatorname{tr} \mathbf{B}_{0}=\frac{1}{\sqrt{p}} \operatorname{tr} \boldsymbol{\Sigma}_{0} \mathbf{H}_{1}-\frac{1}{\sqrt{p}} \operatorname{tr} \boldsymbol{\Sigma}_{0} \mathbf{H}_{0} \\
& \frac{1}{\sqrt{p}} \operatorname{tr} \widehat{\mathbf{\Sigma}}_{0} \mathbf{H}_{i}=\frac{1}{\sqrt{p}} \operatorname{tr} \frac{1}{n_{0}} \sum_{k=1}^{n_{0}} \mathbf{y}_{0 k} \mathbf{y}_{0 k}^{T}[\frac{\alpha_{i}}{n_{0}} \mathbf{y}_{0 j} \mathbf{y}_{0 j}^{T}+\underbrace{\frac{\alpha_{i}}{n_{0}} \sum_{k \neq j} \mathbf{y}_{0 k} \mathbf{y}_{0 k}^{T}+\beta_{i} \widehat{\mathbf{\Sigma}}_{1}+(1-\gamma) \mathbf{I}_{p}}_{\mathbf{A}_{i}}]^{-1} \\
& =\frac{1}{\sqrt{p}} \operatorname{tr} \frac{1}{n_{0}} \sum_{k=1}^{n_{0}} \mathbf{y}_{0 k} \mathbf{y}_{0 k}^{T}\left(\mathbf{A}_{i}^{-1}-\frac{\frac{\alpha_{i}}{n_{0}} \mathbf{A}_{i}^{-1} \mathbf{y}_{0 k} \mathbf{y}_{0 k}^{T} \mathbf{A}_{i}^{-1}}{1+\frac{\alpha_{i}}{n_{0}} \mathbf{y}_{0 k}^{T} \mathbf{A}_{i}^{-1} \mathbf{y}_{0 k}}\right) \\
& =\frac{1}{\sqrt{p}} \operatorname{tr} \mathbf{y}_{0 k}^{T} \mathbf{A}_{i}^{-1} \mathbf{y}_{0 k}-\frac{1}{\sqrt{p}} \operatorname{tr} \frac{\frac{\alpha_{i}}{n_{0}} \mathbf{y}_{0 k}^{T} \mathbf{A}_{i}^{-1} \mathbf{y}_{0 k} \mathbf{y}_{0 k}^{T} \mathbf{A}_{i}^{-1} \mathbf{y}_{0 k}}{1+\frac{\alpha_{i}}{n_{0}} \mathbf{y}_{0 k}^{T} \mathbf{A}_{i}^{-1} \mathbf{y}_{0 k}} \\
& =\frac{1}{\sqrt{p}} \operatorname{tr} \boldsymbol{\Sigma}_{0} \mathbf{H}_{i}-\frac{\alpha_{i}}{\sqrt{p}} \frac{\left(\operatorname{tr} \boldsymbol{\Sigma}_{0} \mathbf{H}_{i}\right)^{2}}{n_{0}+\alpha_{i} \operatorname{tr} \boldsymbol{\Sigma}_{0} \mathbf{H}_{i}}+o(1)
\end{aligned}
$$

Therefore, we can get

$$
\frac{1}{\sqrt{p}} \operatorname{tr} \boldsymbol{\Sigma}_{0} \mathbf{H}_{i}=\frac{1}{\sqrt{p}} \frac{\operatorname{tr} \widehat{\boldsymbol{\Sigma}}_{0} \mathbf{H}_{i}}{1-\frac{\alpha_{i}}{n_{0}} \operatorname{tr} \widehat{\boldsymbol{\Sigma}}_{0} \mathbf{H}_{i}}+o(1)
$$

Finally,

$$
\begin{aligned}
\frac{1}{\sqrt{p}} \operatorname{tr} \mathbf{B}_{0} & =\frac{1}{\sqrt{p}} \operatorname{tr} \boldsymbol{\Sigma}_{0} \mathbf{H}_{1}-\frac{1}{\sqrt{p}} \operatorname{tr} \boldsymbol{\Sigma}_{0} \mathbf{H}_{0} \\
& =\frac{1}{\sqrt{p}} \frac{\operatorname{tr} \widehat{\boldsymbol{\Sigma}}_{0} \mathbf{H}_{1}}{1-\frac{\alpha_{1}}{n_{0}} \operatorname{tr} \widehat{\mathbf{\Sigma}}_{0} \mathbf{H}_{1}}-\frac{1}{\sqrt{p}} \frac{\operatorname{tr} \widehat{\boldsymbol{\Sigma}}_{0} \mathbf{H}_{0}}{1-\frac{\alpha_{0}}{n_{0}} \operatorname{tr} \widehat{\boldsymbol{\Sigma}}_{0} \mathbf{H}_{0}}+o(1)
\end{aligned}
$$


Moreover, since we have $\alpha_{i}=\beta_{1-i}$, the general expression for $\frac{1}{\sqrt{p}} \operatorname{tr} \mathbf{B}_{i}$ is given by

$$
\frac{1}{\sqrt{p}} \operatorname{tr} \mathbf{B}_{i}-\left(\frac{1}{\sqrt{p}} \frac{\operatorname{tr} \widehat{\boldsymbol{\Sigma}}_{i} \mathbf{H}_{1}}{1-\frac{\beta_{i}}{n_{i}} \operatorname{tr} \widehat{\boldsymbol{\Sigma}}_{i} \mathbf{H}_{1}}-\frac{1}{\sqrt{p}} \frac{\operatorname{tr} \widehat{\boldsymbol{\Sigma}}_{i} \mathbf{H}_{0}}{1-\frac{\beta_{1-i}}{n_{i}} \operatorname{tr} \widehat{\boldsymbol{\Sigma}}_{i} \mathbf{H}_{0}}\right) \stackrel{p}{\rightarrow} 0
$$

\section{B.0.3 Proof of $B .3$}

Similarly, the proof for $\frac{1}{p} \operatorname{tr} \mathbf{B}_{i}^{2}$ involves nothing new but the same application of trace lemma and inversion lemma. However, the computations included are more complex. As derived before,

$$
\frac{1}{p} \operatorname{tr} \mathbf{B}_{i}^{2}==\frac{1}{p} \operatorname{tr} \boldsymbol{\Sigma}_{i} \mathbf{H}_{1} \boldsymbol{\Sigma}_{i} \mathbf{H}_{1}-\frac{2}{p} \operatorname{tr} \boldsymbol{\Sigma}_{i} \mathbf{H}_{0} \boldsymbol{\Sigma}_{i} \mathbf{H}_{1}+\frac{1}{p} \operatorname{tr} \boldsymbol{\Sigma}_{i} \mathbf{H}_{0} \boldsymbol{\Sigma}_{i} \mathbf{H}_{0}
$$

We begin with treating case of $i=1$ and $i=0$ just follows the same procedures.

$$
\begin{aligned}
& \frac{1}{p} \operatorname{tr} \widehat{\boldsymbol{\Sigma}}_{1} \mathbf{H}_{1} \widehat{\boldsymbol{\Sigma}}_{1} \mathbf{H}_{1}=\frac{1}{p} \sum_{j=1}^{n_{1}-1} \sum_{k=1}^{n_{1}-1} \operatorname{tr} \frac{\widetilde{\mathbf{y}}_{1, j} \widetilde{\mathbf{y}}_{1, j}^{T}}{n_{1}-1} \mathbf{H}_{1} \frac{\widetilde{\mathbf{y}}_{1, k} \widetilde{\mathbf{y}}_{1, k}^{T}}{n_{1}-1} \mathbf{H}_{1} \\
& =\frac{1}{p} \sum_{j=1}^{n_{1}-1} \sum_{k \neq j} \operatorname{tr} \frac{\widetilde{\mathbf{y}}_{1, j} \widetilde{\mathbf{y}}_{1, j}^{T}}{n_{1}-1} \mathbf{H}_{1} \frac{\widetilde{\mathbf{y}}_{1, k} \widetilde{\mathbf{y}}_{1, k}^{T}}{n_{1}-1} \mathbf{H}_{1}+\frac{1}{p} \sum_{j=1}^{n_{1}-1} \operatorname{tr} \frac{\widetilde{\mathbf{y}}_{1, j} \widetilde{\mathbf{y}}_{1, j}^{T}}{n_{1}-1} \mathbf{H}_{1} \frac{\widetilde{\mathbf{y}}_{1, j} \widetilde{\mathbf{y}}_{1, j}^{T}}{n_{1}-1} \mathbf{H}_{1}
\end{aligned}
$$

Using the inversion lemma, we handle the first term in the previous equation as follows

$\frac{1}{p} \sum_{j=2}^{n_{1}-1} \sum_{k \neq j} \operatorname{tr} \frac{\widetilde{\mathbf{y}}_{1, j} \widetilde{\mathbf{y}}_{1, j}^{T}}{n_{1}-1} \mathbf{H}_{1} \frac{\widetilde{\mathbf{y}}_{1, k} \widetilde{\mathbf{y}}_{1, k}^{T}}{n_{1}-1} \mathbf{H}_{1}=\frac{1}{p} \sum_{j=2}^{n_{1}-1} \sum_{k \neq j} \frac{\left(\frac{1}{n_{1}-1} \widetilde{\mathbf{y}}_{1, j}^{T} \mathbf{H}_{1, j, k} \widetilde{\mathbf{y}}_{1, k}\right)^{2}}{\left(1+\frac{\beta_{1}}{n_{1}-1} \widetilde{\mathbf{y}}_{1, j}^{T} \mathbf{H}_{1, j} \widetilde{\mathbf{y}}_{1, j}\right)^{2}\left(1+\frac{\beta_{1}}{n_{1}} \widetilde{\mathbf{y}}_{1, k} \mathbf{H}_{1, j, k} \widetilde{\mathbf{y}}_{1, k}\right)^{2}}$

where

$$
\mathbf{H}_{1, j}=\left((1-\gamma) \mathbf{I}_{p}+\alpha_{1} \widehat{\boldsymbol{\Sigma}}_{0}+\beta_{1} \widehat{\boldsymbol{\Sigma}}_{1}-\frac{\beta_{1}}{n_{1}-1} \widetilde{\mathbf{y}}_{1, j} \widetilde{\mathbf{y}}_{1, j}^{T}\right)^{-1}
$$

and

$$
\mathbf{H}_{1, j, k}=\left((1-\gamma) \mathbf{I}_{p}+\alpha_{1} \widehat{\boldsymbol{\Sigma}}_{0}+\beta_{1} \widehat{\boldsymbol{\Sigma}}_{1}-\frac{\beta_{1}}{n_{1}-1} \widetilde{\mathbf{y}}_{1, j} \widetilde{\mathbf{y}}_{1, j}^{T}-\frac{\beta_{1}}{n_{1}-1} \widetilde{\mathbf{y}}_{1, k} \widetilde{\mathbf{y}}_{1, k}^{T}\right)^{-1}
$$


. Then applying the trace lemma, we can get the deterministic equivalence as follows

$$
\frac{1}{p} \sum_{j=2}^{n_{1}-1} \sum_{k \neq j} \operatorname{tr} \frac{\widetilde{\mathbf{y}}_{1, j} \widetilde{\mathbf{y}}_{1, j}^{T}}{n_{1}-1} \mathbf{H}_{1} \frac{\widetilde{\mathbf{y}}_{1, k} \widetilde{\mathbf{y}}_{1, k}^{T}}{n_{1}-1} \mathbf{H}_{1}=\frac{n_{1}}{p} \frac{\left(\frac{1}{n_{1}-1} \operatorname{tr} \boldsymbol{\Sigma}_{\mathbf{1}} \mathbf{H}_{1} \boldsymbol{\Sigma}_{\mathbf{1}} \mathbf{H}_{1}\right)}{\left(1+\frac{\beta_{1}}{n_{1}-1} \operatorname{tr} \boldsymbol{\Sigma}_{1} \mathbf{H}_{1}\right)^{4}}+o(1) .
$$

Using similar steps, the second term can be approximated as follows

$$
\frac{1}{p} \sum_{j=1}^{n_{1}-1} \operatorname{tr} \frac{\widetilde{\mathbf{y}}_{1, j} \widetilde{\mathbf{y}}_{1, j}^{T}}{n_{1}-1} \mathbf{H}_{1} \frac{\widetilde{\mathbf{y}}_{1, j} \widetilde{\mathbf{y}}_{1, j}^{T}}{n_{1}-1} \mathbf{H}_{1}=\frac{n_{i}}{p} \frac{\left(\frac{1}{n_{1}-1} \operatorname{tr} \boldsymbol{\Sigma}_{1} \mathbf{H}_{1}\right)^{2}}{\left(1+\frac{\beta_{1}}{n_{1}-1} \operatorname{tr} \boldsymbol{\Sigma}_{1} \mathbf{H}_{1}\right)^{2}}+o(1) .
$$

We thus obtain

$$
\frac{1}{p} \operatorname{tr} \widehat{\boldsymbol{\Sigma}}_{1} \mathbf{H}_{1} \widehat{\boldsymbol{\Sigma}}_{1} \mathbf{H}_{1}=\frac{n_{1}}{p} \frac{\left(\frac{1}{n_{1}-1} \operatorname{tr} \boldsymbol{\Sigma}_{1} \mathbf{H}_{1} \boldsymbol{\Sigma}_{1} \mathbf{H}_{1}\right)}{\left(1+\frac{\beta_{1}}{n_{1}-1} \operatorname{tr} \boldsymbol{\Sigma}_{1} \mathbf{H}_{1}\right)^{4}}+\frac{n_{1}}{p} \frac{\left(\frac{1}{n_{1}-1} \operatorname{tr} \boldsymbol{\Sigma}_{1} \mathbf{H}_{1}\right)^{2}}{\left(1+\frac{\beta_{1}}{n_{1}-1} \operatorname{tr} \boldsymbol{\Sigma}_{1} \mathbf{H}_{1}\right)^{2}}+o(1) .
$$

The remaining terms $\frac{1}{p} \operatorname{tr} \boldsymbol{\Sigma}_{1} \mathbf{H}_{0} \boldsymbol{\Sigma}_{1} \mathbf{H}_{0}$ and $\frac{1}{p} \operatorname{tr} \boldsymbol{\Sigma}_{1} \mathbf{H}_{1} \boldsymbol{\Sigma}_{1} \mathbf{H}_{0}$ can be approximated using exactly the same steps. We have the following results for the remaining two terms.

$$
\begin{aligned}
& \frac{1}{p} \operatorname{tr} \widehat{\boldsymbol{\Sigma}}_{1} \mathbf{H}_{0} \widehat{\boldsymbol{\Sigma}}_{1} \mathbf{H}_{0}=\frac{n_{1}}{p} \frac{\left(\frac{1}{n_{1}-1} \operatorname{tr} \boldsymbol{\Sigma}_{1} \mathbf{H}_{0} \boldsymbol{\Sigma}_{1} \mathbf{H}_{0}\right)}{\left(1+\frac{\beta_{0}}{n_{1}-1} \operatorname{tr} \boldsymbol{\Sigma}_{1} \mathbf{H}_{0}\right)^{4}}+\frac{n_{1}}{p} \frac{\left(\frac{1}{n_{1}-1} \operatorname{tr} \boldsymbol{\Sigma}_{1} \mathbf{H}_{0}\right)^{2}}{\left(1+\frac{\beta_{0}}{n_{1}-1} \operatorname{tr} \boldsymbol{\Sigma}_{1} \mathbf{H}_{0}\right)^{2}}+o(1) \\
& \frac{1}{p} \operatorname{tr} \widehat{\boldsymbol{\Sigma}}_{1} \mathbf{H}_{1} \widehat{\boldsymbol{\Sigma}}_{1} \mathbf{H}_{0}=\frac{n_{1}}{p} \frac{\left(\frac{1}{n_{1}-1} \operatorname{tr} \boldsymbol{\Sigma}_{1} \mathbf{H}_{1} \boldsymbol{\Sigma}_{1} \mathbf{H}_{0}\right)}{\left(1+\frac{\beta_{0}}{n_{1}-1} \operatorname{tr} \boldsymbol{\Sigma}_{1} \mathbf{H}_{0}\right)^{2}\left(1+\frac{\beta_{1}}{n_{1}-1} \operatorname{tr} \boldsymbol{\Sigma}_{1} \mathbf{H}_{1}\right)^{2}} \\
&+\frac{n_{1}}{p} \frac{\left(\frac{1}{n_{1}-1} \operatorname{tr} \boldsymbol{\Sigma}_{1} \mathbf{H}_{0} \frac{1}{n_{1}-1} \operatorname{tr} \boldsymbol{\Sigma}_{1} \mathbf{H}_{1}\right)}{\left(1+\frac{\beta_{0}}{n_{1}-1} \operatorname{tr} \boldsymbol{\Sigma}_{1} \mathbf{H}_{0}\right)\left(1+\frac{\beta_{1}}{n_{1}-1} \operatorname{tr} \boldsymbol{\Sigma}_{1} \mathbf{H}_{1}\right)}+o(1)
\end{aligned}
$$

Now we can solve for $\frac{1}{p} \operatorname{tr} \mathbf{B}_{i}^{2}$.

$$
\begin{aligned}
\frac{1}{p} \operatorname{tr} \boldsymbol{\Sigma}_{1} \mathbf{H}_{1} \boldsymbol{\Sigma}_{1} \mathbf{H}_{1} & =\left(1+\frac{\beta_{1}}{n_{1}-1} \operatorname{tr} \boldsymbol{\Sigma}_{1} \mathbf{H}_{1}\right)^{4} \frac{1}{p} \operatorname{tr} \widehat{\boldsymbol{\Sigma}}_{1} \mathbf{H}_{1} \widehat{\boldsymbol{\Sigma}}_{1} \mathbf{H}_{1} \\
& -\frac{n_{1}}{p}\left(\frac{1}{n_{1}} \operatorname{tr} \boldsymbol{\Sigma}_{1} \mathbf{H}_{1}\right)^{2}\left(1+\frac{\beta_{1}}{n_{1}-1} \operatorname{tr} \boldsymbol{\Sigma}_{1} \mathbf{H}_{1}\right)^{2}+o(1) .
\end{aligned}
$$




$$
\begin{aligned}
\frac{1}{p} \operatorname{tr} \boldsymbol{\Sigma}_{1} \mathbf{H}_{0} \boldsymbol{\Sigma}_{1} \mathbf{H}_{0} & =\left(1+\frac{\beta_{0}}{n_{1}-1} \operatorname{tr} \boldsymbol{\Sigma}_{1} \mathbf{H}_{0}\right)^{4} \frac{1}{p} \operatorname{tr} \widehat{\boldsymbol{\Sigma}}_{1} \mathbf{H}_{0} \widehat{\boldsymbol{\Sigma}}_{1} \mathbf{H}_{0} \\
& -\frac{n_{1}}{p}\left(\frac{1}{n_{1}} \operatorname{tr} \boldsymbol{\Sigma}_{1} \mathbf{H}_{0}\right)^{2}\left(1+\frac{\beta_{0}}{n_{1}-1} \operatorname{tr} \boldsymbol{\Sigma}_{1} \mathbf{H}_{0}\right)^{2}+o(1) . \\
\frac{1}{p} \operatorname{tr} \boldsymbol{\Sigma}_{1} \mathbf{H}_{1} \boldsymbol{\Sigma}_{1} \mathbf{H}_{0} & =\left(1+\frac{\beta_{1}}{n_{1}-1} \operatorname{tr} \boldsymbol{\Sigma}_{1} \mathbf{H}_{1}\right)^{2}\left(1+\frac{\beta_{0}}{n_{1}-1} \operatorname{tr} \boldsymbol{\Sigma}_{1} \mathbf{H}_{0}\right)^{2} \frac{1}{p} \operatorname{tr} \widehat{\boldsymbol{\Sigma}}_{1} \mathbf{H}_{1} \widehat{\boldsymbol{\Sigma}}_{1} \mathbf{H}_{0} \\
- & \frac{n_{1}}{p}\left(\frac{1}{n_{1}} \operatorname{tr} \boldsymbol{\Sigma}_{1} \mathbf{H}_{1}\right)\left(\frac{1}{n_{1}} \operatorname{tr} \boldsymbol{\Sigma}_{1} \mathbf{H}_{0}\right)\left(1+\frac{\beta_{1}}{n_{1}-1} \operatorname{tr} \boldsymbol{\Sigma}_{1} \mathbf{H}_{1}\right) \\
& \left(1+\frac{\beta_{0}}{n_{1}-1} \operatorname{tr} \boldsymbol{\Sigma}_{1} \mathbf{H}_{0}\right)+o(1) .
\end{aligned}
$$

Finally, adding these three terms together,

$$
\begin{aligned}
\frac{1}{p} \operatorname{tr} \mathbf{B}_{1}^{2} & =\left(1+\frac{\beta_{1}}{n_{1}-1} \operatorname{tr} \boldsymbol{\Sigma}_{1} \mathbf{H}_{1}\right)^{4} \frac{1}{p} \operatorname{tr} \widehat{\boldsymbol{\Sigma}}_{1} \mathbf{H}_{1} \widehat{\boldsymbol{\Sigma}}_{1} \mathbf{H}_{1}-\frac{n_{1}}{p}\left(\frac{1}{n_{1}} \operatorname{tr} \boldsymbol{\Sigma}_{1} \mathbf{H}_{1}\right)^{2}\left(1+\frac{\beta_{1}}{n_{1}-1} \operatorname{tr} \boldsymbol{\Sigma}_{1} \mathbf{H}_{1}\right)^{2} \\
& +\left(1+\frac{\beta_{0}}{n_{1}-1} \operatorname{tr} \boldsymbol{\Sigma}_{1} \mathbf{H}_{0}\right)^{4} \frac{1}{p} \operatorname{tr} \widehat{\boldsymbol{\Sigma}}_{1} \mathbf{H}_{0} \widehat{\boldsymbol{\Sigma}}_{1} \mathbf{H}_{0}-\frac{n_{1}}{p}\left(\frac{1}{n_{1}} \operatorname{tr} \boldsymbol{\Sigma}_{1} \mathbf{H}_{0}\right)^{2}\left(1+\frac{\beta_{0}}{n_{1}-1} \operatorname{tr} \boldsymbol{\Sigma}_{1} \mathbf{H}_{0}\right)^{2} \\
& -2\left(1+\frac{\beta_{1}}{n_{1}-1} \operatorname{tr} \boldsymbol{\Sigma}_{1} \mathbf{H}_{1}\right)^{2}\left(1+\frac{\beta_{0}}{n_{1}-1} \operatorname{tr} \boldsymbol{\Sigma}_{1} \mathbf{H}_{0}\right)^{2} \frac{1}{p} \operatorname{tr} \widehat{\boldsymbol{\Sigma}}_{1} \mathbf{H}_{1} \widehat{\boldsymbol{\Sigma}}_{1} \mathbf{H}_{0} \\
& +\frac{2 n_{1}}{p}\left(\frac{1}{n_{1}} \operatorname{tr} \boldsymbol{\Sigma}_{1} \mathbf{H}_{1}\right)\left(\frac{1}{n_{1}} \operatorname{tr} \boldsymbol{\Sigma}_{1} \mathbf{H}_{0}\right)\left(1+\frac{\beta_{1}}{n_{1}-1} \operatorname{tr} \boldsymbol{\Sigma}_{1} \mathbf{H}_{1}\right)\left(1+\frac{\beta_{0}}{n_{1}-1} \operatorname{tr} \boldsymbol{\Sigma}_{1} \mathbf{H}_{0}\right)
\end{aligned}
$$




\section{Papers Submitted and Under Preparation}

- Xiaoke Yang, Khalil Elkhalil, Abla Kammoun, Tareq Y. Al-Naffouri and MohamedSlim Alouini "Regularized Discriminant Analysis: A Large Dimensional Study", Accepted in ISIT, Mar. 2018. 